\title{
Res et signa. Formen der Ambiguität in der Malerei des Cinquecento
}

\section{Denken des Malens}

Die Analogie von Malen und Denken, von Kunst und Philosophie ist in der Renaissance oft beschworen worden. Behauptet Giordano Bruno in seiner Explicatio triginti sigillorum von 1583 kühn, dass „derjenige kein Philosoph sei, der nicht bilde und male" , so beschwört Leone Battista Alberti bereits im Jahre 1435 in seiner kunsttheoretischen Gründungsschrift, dem Traktat Della pittura, den philosophischen und wissenschaftlichen Anspruch der Malerei im Vorstellungsbild der „piú grassa Minerva“. ${ }^{2}$ Die damit verbundene Absicht im Hinblick auf den geforderten Status der Bildkünste, die sich wissenschaftliches Profil geben wollten ${ }^{3}$ und somit Rang und Status von „freien“ Künsten beanspruchten, ist oft betont worden. Jenseits von solchen Absichten deuten Albertis und Brunos Aussagen aber auch auf das Vermögen zur Reflexion der produktiven Bedingungen und Möglichkeiten der Philosophie und Malerei und der ihnen eigenen Diskursivität hin.

Die reziproke Erweiterung des metaphorischen Tagungstitels „Kann das Denken malen?" um die Formel vom "Denken des Malens" scheint auf den ersten Blick eine banale Selbstverständlichkeit zu formulieren. Dass die Entwicklung von Bildideen und -konzeptionen und die Überlegungen bezüglich ihrer Realisationen mit den jeweiligen Mitteln der Künste geistige Leistungen der Künstler sind, wird niemand ernsthaft bestreiten wollen, auch nicht, dass diese dabei „malend“ oder „bildend“ zu einer ganzen Reihe von Diskursen - in der Frühen Neuzeit sind dies vorrangig theologische, künstlerische bzw. kunsttheoretische und (natur)philoso-

* Für wichtige Hinweise und Hilfen danke ich Christoph Thoenes, Fosca Mariani Zini, Marianne Koos, Stefan Koldehoff und Nadja Horsch.

1 ,ideoque philosophi sunt quodammodo pictores atque poëtae, poëtae pictores et philosophi, pictores philosophi et poëtae, mutuoque veri poëtae, veri pictores et veri philosophi se diligunt et admirantur; non est enim philosophus, nisi qui fingit et pingit" (Giordano Bruno, Explicatio tringinta sigillorum, Opera latine conscripta, hrsg. v. F. Fiorentino u. a., Neapel/Florenz 1879-91, Facs.-Neudr. Stuttgart 1962, II/2, S. 133 [Hervorh. d. Verf.in]; vgl. auch den Beitrag von Thomas Leinkauf in diesem Band.

2 Leone Battista Alberti, Della pittura. Über die Malkunst, I1, hrsg., eingel., übers. u. komm. v. Oskar Bätschmann, Sandra Gianfreda, Darmstadt 2002, S. 66.

3 Vorrangig zu nennen ist hier die Entwicklung der Zentralperspektive, die „wissenschaftlich“ abgesichert wurde; siehe hierzu Frank Büttner, „Rationalisierung der Mimesis: Anfänge der konstruierten Perspektive bei Brunelleschi und Alberti“, in: Andreas Kablitz/Gerhard Neumann (Hrsg.), Mimesis und Simulation, Freiburg 1998, S. 55-87; Hubert Damisch, L'origine de la perspective, Paris ${ }^{3} 1993$. 
phische - Stellung nehmen. Was gleichwohl verschieden beantwortet wird, sind die Fragen nach der Intensität und Intentionalität in dieser Partizipation und nach der Art und Weise des Reflexionsvermögens der Bildkünste, ihrer Bildlogik und spezifischen Theoriehaltigkeit, die in der Forschung sowohl in systematischer als auch in historischer Perspektive - vorrangig am Einzelbeispiel - diskutiert wurden. ${ }^{4}$ Welcher Art ist also dieses „denkende Malen“ im Unterschied zum diskursiven Vermögen der Philosophie, das in der Renaissance für sich in Anspruch nimmt, sich auf das Bildhafte hin zu öffnen?

Ich möchte diese Frage im Folgenden mit Bezug auf einen begrenzten Gegenstand verfolgen, und dabei ein Thema behandeln, das im Denken der Renaissance generell eine große Rolle spielt und das Rahmenthema dieses Bandes bezüglich der Wechselwirkung von Philosophie und Malerei in der Renaissance im Kern trifft: die Generierung von „Inhalt“ bzw. „Bedeutung“ im Prozess der Signifikation, oder mit den Worten Lorenzo Vallas, das Verhältnis von res und signa. ${ }^{5}$

Auf theoretischer Ebene ist dieses für die Sprach- und Bildkünste so zentrale Thema in der Philosophie der Zeit ungleich ausführlicher behandelt worden als in der Kunst, weshalb es sinnvoll ist, in knapper Form die Grundzüge der sprachphilosophischen Überlegungen der Renaissance zu skizzieren, um daran anschließend die Verbindung zur Malerei aufzuzeigen.

Was das Denken und Schreiben der Sprachhumanisten wie Lorenzo Valla, Rudolph Agricola sowie bereits Petrarca eint, ist eine Sicht auf die Welt "nicht als Welt der seienden, sondern als Welt der zu Wort gekommenen Dinge" ${ }^{\text {"6 }}$. Nach ihrer Auffassung „begegnet der Mensch“, wie es Stephan Otto formuliert, „nicht einer objektiv seienden Dingwelt, die er nachträglich mit Hilfe von Sprachzeichen benennt, sondern er bemächtigt sich der Dinge im subjektiven Medium der Sprache selber. "7 Damit lösen sich die genannten Denker von der Ontologie der Scholastik und knüpfen an die die aristotelische Philosophie tragende Annahme einer Entsprechung von Sprache und Sein an. Hierdurch ergibt sich der Bezug zur Rhetorik, die bei Aristoteles ebenso wie im denkgeschichtlichen Horizont der Renaissance weit mehr ist als die Kunst des überzeugenden und guten Sprechens, vielmehr als „Organon der Wahrheitsfindung". (Otto) gilt. So schreibt Lorenzo Valla in seinen Dialecticae disputationes:

4 Aus der umfangreichen Literatur seinen nur wenige Titel genannt: Christa Maar/Hubert Burda (Hrsg.), Iconic Turn. Die neue Macht der Bilder, Köln 2004; Ron Burnett, How Images Think, Cambridge (MA) 2004; Valeska von Rosen/Klaus Krüger/Rudolf Preimesberger (Hrsg.), Der stumme Diskurs der Bilder. Reflexionsformen des Ästhetischen in der Kunst der frühen Neuzeit, München/Berlin 2003; Gottfried Boehm (Hrsg.), Was ist ein Bild? München ${ }^{2} 1995$.

5 Siehe hierzu Anm. 8.

6 Stephan Otto, Renaissance und frühe Neuzeit (Geschichte der Philosophie in Text und Darstellung 3), Stuttgart 1984, S. 99 (ohne die Hervorh. d. Autors). Ich beziehe mich hier wie im Folgenden eng auf die Schriften Stephan Ottos; siehe auch ders., „Rhetorische Techne oder Philosophie sprachlicher Darstellungskraft?", in: Zeitschrift für philosophische Forschung 37 (1983), S. 497-514; ders., Das Wissen des Ähnlichen. Michel Foucault und die Renaissance, Frankfurt a. M. 1992; ders., Giambattista Vico, Grundzüge seiner Philosophie, Stuttgart 1989.

7 Otto (1984), a. O. Anm. 6, S. 99. 
Et apud rhetores: quod omnis oratio necesse est habeat \& rem \& uerba: [...] Que uerba quidê apellantes signa dicût omnia côstare, è rebus \& signis.

Bei den Rhetorikern heißt es, daß [...] die Rede als solche ihren Bestand hat aus dem, was bezeichnet wird, und aus dem, was bezeichnet: aus Sachen also und aus Wörtern. Indem die Rhetoriker diese Wörter nun ,Zeichen' nennen, behaupten sie: Alles besteht aus Sachen und Zeichen. ${ }^{8}$

Damit ist die Benennbarkeit der Dinge kraft der inneren Bedeutungsrelation der verba bzw. signa zu den res angesprochen, und dies ruft generell den Themenkomplex der Darstellbarkeit auf. Um sie kreist die Sprachdialektik des RenaissanceHumanismus, und dies wesentlich mit dem Fokus auf der Darstellungskraft bzw. -fähigkeit der Sprache. Nur ein Beispiel von Lorenzo Valla sei genannt: Er argumentiert, wenn das Wort „Holz" dem hölzernen Ding zukommen können soll, muss eine Ähnlichkeitsbeziehung zwischen Sprache und Sache bestehen, ${ }^{9}$ denn es sei die Sprache, welche die Entsprechung von Wort und Wirklichkeit erzeuge. Bekanntlich hat die Kategorie der „Ähnlichkeit“ in jüngerer Zeit dadurch Konjunktur, dass Michel Foucault sie in seiner Ordnung der Dinge zur Episteme der Renaissance erhob. ${ }^{10}$ Im Rahmen dieses Bandes ist die solchen Überlegungen verknüpfte Metaphorik des Visuellen, die auf die Bedeutung der Bildhaftigkeit von Sprache und Denken hinweist, aufschlussreich.

Was die Bezeichnungsfrage in den Bildkünsten angeht, sind die Voraussetzungen natürlich andere, da diese nicht mit dem Problem der Arbitrarität der (sprachlichen) Zeichen konfrontiert sind. Was aber die Adäquanz von res und signa, des Bezeichneten und des Bezeichnenden, für die Sprach- und Bildkünste der Frühen Neuzeit gleichermaßen virulent macht, ist die Kategorie der Angemessenheit - bekanntlich in der Frühen Neuzeit mit ihrem rhetorischen Kunstsystem die Leitkategorie poetologischer und kunsttheoretischer Reflexion. Ich will dieses Thema im Folgenden nicht auf theoretischer Ebene verhandeln und untersuchen, wie die Reflexion der Künste im Medium der Sprache, die uns in der Renaissance erstmals mit Albertis Libro della pittura greifbar wird, dieses Thema verhandelte, und worin dabei die Bezüge zur Sprachdialektik der Renaissance-Humanisten liegen; ich möchte dies vielmehr konkret mit Bezug auf Bilder tun, um auf diese Weise dem

8 Lorenzo Valla, „Dialecticae disputationes“, in: Opera omnia, hg. u. eingel. v. Eugenio Garin, Turin 1962, I \$2. S. 648; dt. Übers. von Otto (1984), a. O. Anm. 6, S. 108.

9 Valla, ebd. I \$14, S. 676: „Postremo inuentae sunt literae quasi mutae uoces, siue uocû imagines: ut ipse uoces sunt quasi imagines significationû quae iam proprie dicûtur uocabula. Atq hoc est quicquid loquimur: etiâ ipsum substantia, qualitas, actio:atq adeo ipsum res. Nam sicuti ligno nomen lignum est, \& lapidi lapis, \& ferro ferrum“; dt. Übers. v. Otto (1984), S. 111: „Schließlich sind die Schriftzeichen als gleichsam stumme Sprachausdrücke oder als Abbilder stimmhafter Sprache erfunden worden; auch die stimmhaften Wörter selbst sind sozusagen Abbilder von Bedeutungen als die sie nun Wörter im eigentlichen Sinne genannt werden. Und genau das ist es, was wir aussprechen - und eben dies gilt auch für ,Substanz', ,Qualität', ,Handlung', und im selben Maße gilt es für ,Saché. Denn wie dem Holz der Name Holz zukommt, so auch dem Stein der Name Stein und dem Eisen der Name Eisen.“

10 Michel Foucault, Les mots et les choses, Paris 1966; hierzu grundlegend Otto (1992), a. O. Anm. 6. 
spezifischen „Denken der Malerei“ näher zu kommen. Dabei ist meine These, dass bestimmte, im Folgenden näher zu umreißende Phänomene in der Malerei des 16. Jahrhunderts im Kontinuum mit Entwicklungen des Sprachhumanismus stehen, in denen die Reflexion über das Verhältnis von „res et signa“ und die Verweiskraft von (Bild-)Zeichen eine wichtige Rolle spielten. Ich möchte zeigen, dass sich dieses Denken in der Reflexion über die Frage, wie „Bedeutung“ in Bildern entsteht, manifestierte. Wie zu zeigen sein wird, kreiste das Bilddenken speziell der oberitalienischen Maler in der Renaissance um diese Frage intensiv. Dass Künstler über solche Themen nachgedacht haben, ist eine Selbstverständlichkeit - was mich daran interessiert, ist das reflexive Moment dabei, das sich in den Bildern dann niederschlägt, wenn ihre Schöpfer Verfahren und Prozess der Signifikation explizit zum Thema machen. Genau an diesem Punkt wird für uns das Denken der Maler „sichtbar“. Ich wähle bewusst sehr verschieden strukturierte Gemälde, in denen sich die Ambiguität und Arbitrarität divergierend manifestiert, um exemplarisch die Bandbreite der Möglichkeiten, wie sie im 15. und frühen 16. Jahrhundert durchgespielt wurden, aufzuzeigen.

\section{Barbara}

Die Reflexion über res und signa im Bereich der Künste zeigt sich grundsätzlich bereits in der Kodifizierung des diesbezüglichen Wissens in Kompendien mit der Absicht seiner Normierung einerseits und seiner Abrufbarkeit für Künstler und Rezipienten andererseits. Emblembücher und -verzeichnisse, ikonologische Traktate, mythologische und hagiographische Schriften halten das Wissen bezüglich christlicher Ikonographie, antiker Götterwelt und Mythologie, Hieroglyphik und Heraldik bereit und bieten so ein Gerüst visueller Topoi, ${ }^{11}$ das den Künstlern die Informationen liefert, wie ein Sujet überhaupt darzustellen ist. ${ }^{12}$ Gleichzeitig statten sie damit die Rezipienten mit dem nötigen Wissen aus, das ihnen die Identifizierung der Darstellungen und überhaupt die Kommunikation über sie ermöglicht. So war es sicherlich einem großen Teil der über ein gewisses Maß an Bildung verfügenden Betrachter um 1500 geläufig, dass ein Turm in der Hand einer weib-

11 Vgl. Wilhelm Schmidt-Biggemann, „Topische Modelle in Theorie und Praxis der Renaissance“, in: Ulrich Pfisterer/Max Seidel (Hrsg.), Visuelle Topoi. Erfindung und tradiertes Wissen in den Künsten der italienischen Renaissance, München/Berlin 2003, S. 11-20, hier S. 14.

12 Ich nenne nur wenige Beispiele: Andrea Alciatis Emblematum liber (Augsburg 1531); (der Autor hatte bereits 1521 in Mailand eine Emblemsammlung zusammengestellt; siehe Holger Homann, Studien zur Emblematik des 16. Jahrhunderts, Utrecht 1971, S. 25-40), Paolo Giovios, Dialogo dell Imprese Militari et Amorose (Rom 1555), Piero Valerianos Hierogryphica (Basel 1556), Vincenzo Cartaris Imagini delli dei de gl'antichi (Venedig 1557) und Cesare Ripas Iconologia (Rom 1693). Vgl. hierzu Mario Praz, Studies in Seventeenth Century Imagery, London 1939; Arthur Henkel/Albrecht Schöne (Hrsg.), Emblemata. Handbuch zur Sinnbildkunst des XVI. und XVII. Jahrhunderts, Stuttgart 1967. Zum Aspekt der Wissenskodifizierung und des topischen Charakters der Embleme bes. Bernhard F. Scholz, Emblem und Emblempoetik: Historische und systematische Studien, Berlin 2002, S. 43-62. 
Abb. 1: Parmigianino, hl. Barbara; Madrid, Museo del Prado. (Farbtafel 19)

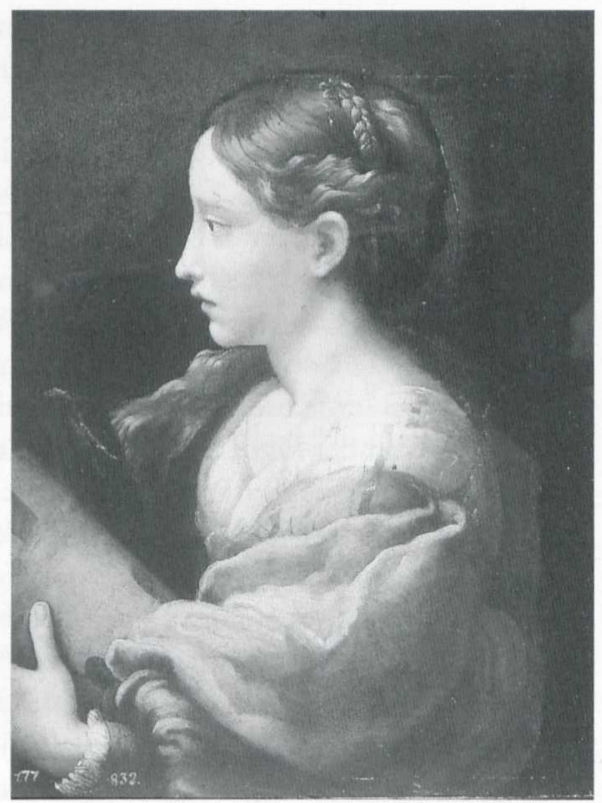

lichen Figur diese als hl. Barbara benennt, oder eine Maske in der Hand eine weibliche Figur als (Allegorie der) Pictura ausweist.

Ich bleibe bei dem Beispiel der hl. Barbara mit ihrem Turm und beziehe mich auf Parmigianinos kleinformatige preziose Darstellung dieser Heiligen von ca. 1522, die sich im Prado befindet und auf der großen Parmigianino-Ausstellung in Parma vor wenigen Jahren einen besonderen Blickfang bildete (Abb. 1). ${ }^{13}$ Dies tat sie aufgrund der malerischen delicatezza der Figur durch die porzellanhafte Wirkung des Inkarnats, die durch den Bildträger Holz in besonderer Weise zur Geltung kommt, durch die virtuose offene Pinselführung auf dem rosafarbenen Gewand, das sich vom dunklen Grün des Hintergrunds effektvoll abhebt, und durch die besondere Anlage der Figur im Bild: Wir sehen die Heilige in Halbfigur, und damit in einem etwas größeren Bildausschnitt als es dem Prototyp solcher „Devotionsbilder“, der Ikone, entspricht - wenn das Bild denn überhaupt als „Devotionsbild" benutzt wurde. Weder wissen wir das in diesem konkreten Fall, noch lässt sich das in einer Zeit, in der private Sammlungen entstehen und damit ästhetische Rezeptionsformen zu den religiösen hinzutreten, diese überlagern oder gar ersetzen

$1348 \times 39 \mathrm{~cm}$ (mit Anstückungen an allen Seiten); Madrid, Museo del Prado; siehe für das Gemälde Sylvia Ferino-Pagden, in: Lucia Fornati/dies. (Hrsg.), Parmigianino und der europäische Manierismus (Ausst.-Kat. Parma/Wien 2003), Cinisello Balsamo 2003, S. 222, Nr. II.2.5.; Mary Vaccaro, Parmigianino: i dipinti, Turin 2002, S. 128 f. Nr. 3. Das Bild ist 1686 im Alcázar in Madrid belegt; möglicherweise ist auch ein Eintrag im Inventar der Sammlung von Alessandro d'Este von 1618 darauf zu beziehen. Hierfür Claudia Cremonini, „Le raccolte d'arte del Cardinale Alessandro d'Este. Vicende collezionistiche tra Modena e Roma“, in: Jadranka Bentini (Hrsg.), Sovrane passioni. Studi sul collezionismo estense, Mailand 1998, S. 91-137, hier Anm. 46, S. 107 f. 
können, generell sagen. Der übliche Terminus „Devotionsbild“ für solche Heiligendarstellungen in Halbfigur, der ja eine spezifische Nutzung suggeriert, taugt also nur als Verständigungshilfe. ${ }^{14}$

Was Parmigianinos Darstellung der hl. Barbara in jedem Fall von einer „Standardformulierung" eines Devotionsbildes unterscheidet und auch eine entsprechende Nutzung unwahrscheinlich macht, ist die Positionierung der Figur im Bild: ihre Drehung zur Seite, durch die uns die Heilige im Profil zugewandt ist, wie es im Übrigen auch in der Gattung des Porträts zu diesem Zeitpunkt längst nicht mehr üblich war. So verzichtet Parmigianino auf ein konstitutionelles und funktionsbedingtes Moment eines „Devotionsbildes“, nämlich die Ausrichtung von Körper und Gesicht der Figur auf ihr "Gegenüber“, ihr „Für-den-Betrachter-Dasein“. ${ }^{15}$ Zugespitzt formuliert wirkt das Bild auch durch die Überschneidung der Hand der Heiligen und des von ihr gehaltenen Gegenstands wie ein Ausschnitt aus einer narrativ strukturierten „storia“" (in der Terminologie Albertis), in die die Heilige eingebunden ist, und auf die sie, wie der minimal geöffnete Mund indiziert, leicht emotionalisiert reagiert. Diese Leseweise wird zwar von dem attributhaft großen Turm in ihren Armen unterlaufen, er ist allerdings auch erst auf den zweiten Blick als solcher erkennbar - ein Effekt, der insbesondere im originalen Zustand der Tafel ohne Anstückungen an allen Seiten noch größer gewesen sein muss. ${ }^{16}$ Auch der überaus schwache Nimbus der Heiligen an ihrem Hinterkopf fällt erst spät ins Auge. Er ist zwar Garant dafür, dass wir die weibliche Figur als die hl. Barbara zu identifizieren haben, doch bleibt augenfällig, dass Parmigianino offenbar alles daran setzte, uns die Erkenntnis bezüglich der Identität der Figur nicht sofort haben zu lassen, unsere Wahrnehmung vielmehr durch die Ungewöhnlichkeit der Bildform und die attraktive Gestaltungsweise von Figur und Bild in andere Bahnen zu lenken.

14 Zum viel bedachten Problemkomplex: Robert Suckale, „Arma Christi. Überlegungen zur Zeichenhaftigkeit mittelalterlicher Andachtsbilder", in: Städel-Jahrbuch 6 (1977), S. 177-208, wiederabgedruckt mit einer Nachschrift, in: ders., Stil und Funktion. Ausgewählte Schriften zur Kunst des Mittelalters, hrsg. v. Peter Schmidt, Gregor Wedekind, München/Berlin 2003; Karl Schade, Andachtsbild. Die Geschichte eines kunsthistorischen Begriffs, Weimar 1996.

15 Vgl. Ferino-Pagden, a. O. Anm. 13, S. 222: „Ihre Wendung ins Profil ist dabei kaum inhaltlich motiviert: sie ist bereits als reine Kunstform aufzufassen."

16 Die Anstückungen sind mit bloßem Auge vor dem Original zu erkennen. Sie verlaufen auf der linken Längsseite unmittelbar neben der Inventarnummer 1; mithin war der größere Teil der charakteristischen zinnenartigen Einbuchtungen des Turm ursprünglich nicht zu erkennen. Möglicherweise war die Anstückung Folge der als störend wahrgenommenen Uneindeutigkeit der Figur. Die bekannten Kopien des Bildes in Chatsworth, Pomona und Den Haag weisen die Erweiterung nicht auf. Bezeichnenderweise ist in der Fassung in Den Haag auch der Nimbus deutlich vergröBert [siehe die Abbildung in Vaccaro, a. O. Anm. 13, S. 129]. 


\section{Sebastian}

Schwieriger den Prozess der Wahrnehmung eines Gemäldes durch einen frühneuzeitlichen Betrachter zu rekonstruieren, fällt es bei einem von der Hand Giorgiones stammenden, mit $48 \times 42 \mathrm{~cm}$ ebenfalls kleinformatigen Gemälde mit einer Halbfigur, dem berühmten Knaben mit Pfeil im Wiener Kunsthistorischen Museum von ca. 1506-08 (Abb. 2). ${ }^{17} \mathrm{Um}$ seine Benennung gibt es in der kunsthistorischen Forschung seit langem Diskussionen: Man hat in ihm sowohl einen hl. Sebastian oder ein Bildnis eines (unbekannten) jungen Mannes in der „Rolle“ des Sebastian erkennen wollen, als auch einen Apoll, Eros oder Paris. ${ }^{18}$ Das Faktum, dass diese Frage so schwer zu entscheiden ist, ist das, worauf es mir hier ankommt. Verweist das Attribut des Knaben, der Pfeil, auf einen „Sebastian“, so wäre eine entsprechende Darstellung des Heiligen ungewöhnlich, und zwar aufgrund der Halbfigur, der all' antica-Kleidung des Knaben, des Fehlens von Nimbus und Wunden und nicht zuletzt auch aufgrund der Attributivität des Pfeils in der Hand des Knaben - bekanntlich wird Sebastian in der Bildtradition dazu benutzt, einen männlichen Akt ins Bild zu setzen, in dessen apollinisch schönem Körper die Pfeile stecken, die dort meist keine entstellende Wirkung haben. ${ }^{19}$ Eine Darstellungsform, wie Giorgione sie wählt, wäre also eine Ausnahmeerscheinung innerhalb der Sebastians-Darstellungen, gleichwohl wäre sie nicht einmalig: Auch Raffaels wenige Jahre zuvor entstandener "Sebastian“ in Halbfigur in der Accademia Carrara in Bergamo (Abb. 3) zeichnet sich dadurch aus, wenngleich der Nimbus der Figur und die kostbarere Gewandung hier die in Giorgiones Bild gänzlich fehlende Aura des Sakralen evozieren. ${ }^{20}$

17 Pappelholz; siehe für das Gemälde Christian Hornig, Giorgiones Spätwerk, München 1987, S. 212 f.; Terisio Pignatti/Filippo Pedrocco, Giorgione, Mailand 1999, S. 132, Nr. 15; Marianne Koos, „Giorgione da Castelfranco. Knabe mit Pfeil“, in: Sylvia Ferino-Pagden/Giovanna Nepi Scirè (Hrsg.), Giorgione. Mythos und Enigma (Ausst.-Kat. Wien 2004), Mailand 2004, S. 184-187; dies., „Amore dolce-amaro. Giorgione und das ideale Knabenbildnis der venezianischen Renaissancemalerei“, in: Marburger Jahrbuch für Kunstwissenschaft 33 (2006), S. 113-174. Ich danke der Autorin, dass sie mir ihr Manuskript vor der Drucklegung zur Verfügung gestellt hat.

18 Eine ausführliche Übersicht über die Forschungsdiskussion in Bezug auf die Identitätsfrage bei Koos (2006), a. O. Anm. 17.

19 Für die Sebastians-Ikonographie siehe: Detlev von Hadeln, Die wichtigsten Darstellungsformen des h. [!] Sebastian in der italienischen Malerei bis zum Ausgang des Quattrocento, Straßburg 1906; Victor Kraehling, Saint Sébastien dans l'art, Paris 1938; Jacques Darriulat, Sebastien. Le Renaissant. Sur le martyre de saint Sébastien dans la deuxième moitié du Quattrocento, Paris 1998; Karim RessouniDemigneux, Saint-Sébastien, Paris 2000; Sebastiaan. Martelaar of mythe, Zwolle 1993; Daniela Bohde, Haut, Fleisch und Farbe. Körperlichkeit und Materialität in den Gemälden Tizians, Emsdetten/Berlin 2002, S. 209-246; dies., „Ein Heiliger der Sodomiten? Das erotische Bild des HI. Sebastian im Cinquecento", in: Mechthild Fend/Marianne Koos (Hrsg.), Männlichkeit im Blick: Visuelle Inszenierungen in der Kunst seit der Frühen Neuzeit, Köln 2004, S. 79-98; Luba Freedman, „Saint Sebastian in Veneto Painting: The ,Signals' Addressed to ,Learned" Spectators", in: Venezia Cinquecento 15 (1988), S. 5-20. Angemerkt sei, dass die all'antica-Kleidung der Figur zwar ungewöhnlich für eine Sebastians-Darstellung wäre, gleichwohl nicht ausgeschlossen ist.

20 45,1 x 36,5 cm; ca. 1501/02; für das Gemälde siehe Sylvia Ferino-Pagden/M. Antonietta Zancan, Raffaello. Catalogo completo dei dipinti, Florenz 1989, S. 18, Nr. 4; Jürg Meyer zur Capellen, 


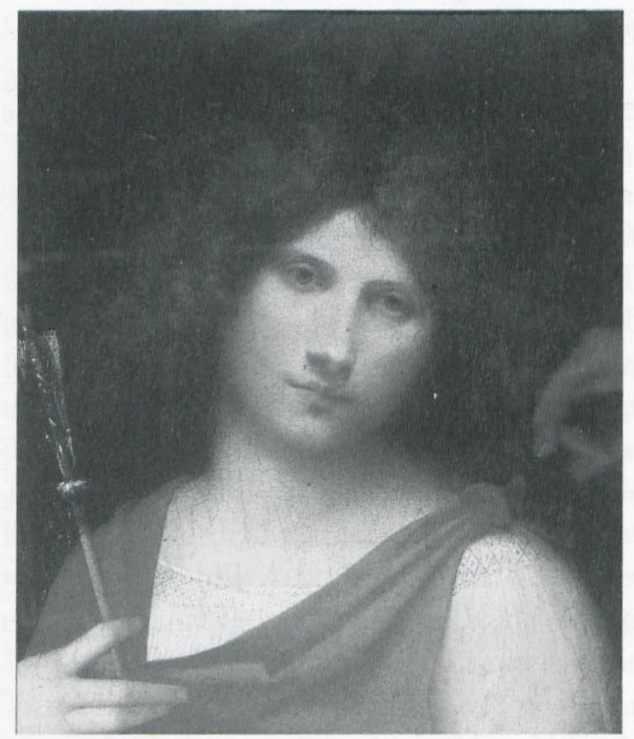

Abb. 2: Giorgione, Knabe mit Pfeil; Wien, Kunsthistorisches Museum. (Farbtafel 20)

Was beide Gemälde miteinander verbindet, ist der Blick der Figur aus dem Bild, bei dem es sich um ein konventionalisiertes Zeichen für ein devotionales Heiligenbild handelt, das insbesondere bei Märtyrer- oder Passionsdarstellungen die emotionale "Teilhabe" der Betrachter sichern soll. Die Frage, um wen es sich bei Giorgiones Knaben handelt, beschäftigte wohl auch bereits die Zeitgenossen des Malers, worauf Marcantonio Michiels frühe Erwähnung des Gemäldes den Hinweis gibt. Er beschreibt das Gemälde unspezifisch als „[... pittura della testa del garzone, che tien in mano la setta" 21 .

Was die Frage der Identifizierung in besonderer Weise erschwert, ist die Neuartigkeit der Darstellung androgyner und oft melancholisch gestimmter Knaben in Halbfigur in der venezianischen Malerei um 1500, bei denen es sich teilweise um Porträts handelt. Marianne Koos hat dieser Bildgattung eine grundlegende Studie gewidmet, in der sie dafür plädiert, auch den Knaben mit Pfeil darunter zu subsumieren und in ihm ein „Bild des profanen Begehrens“ zu erkennen. ${ }^{22}$ In ihrer Lesart steht der Pfeil für den Blick, der zur Liebe erregt und mit den Konnotationen

Raphael. A critical catalogue of his paintings. I The Beginnings in Umbria and Florence, ca. 15001508, Landshut 2001, S. 117-119, Nr. 6; Irene Baldriga, „Raffaello Sanzio. Saint Sébastien“, in: Patrizia Nitti/Marc Restellini (Hrsg.), Raphaël. Grace et Beauté (Ausst.-Kat. Paris 2001/2002), Paris 2001, S. 82-85; Sylvie Béguin/Cristiana Garofalo, Raffaello. Catalogo completo dei dipinti, Santarcangelo di Romagna 2002, S. 17, Nr. 3.

21 Theodor Frimmel, Der Anonimo Morelliano. Marcantonio Michel's Notizia d'opere del disegno, Wien 1888, S. 104.

22 Koos (2006), a. O. Anm. 17, S. 126. Hingewiesen sei auch auf ihre Dissertation: Bildnisse des Begehrens. Das lyrische Männerporträt in der venezianischen Malerei des frühen 16. Jahrhunderts - Giorgione, Tizian und ihr Umkreis, Emsdetten/Berlin 2006. 
Abb. 3: Raffael, hl. Sebastian; Bergamo, Accademia Carrara. (Farbtafel 21)

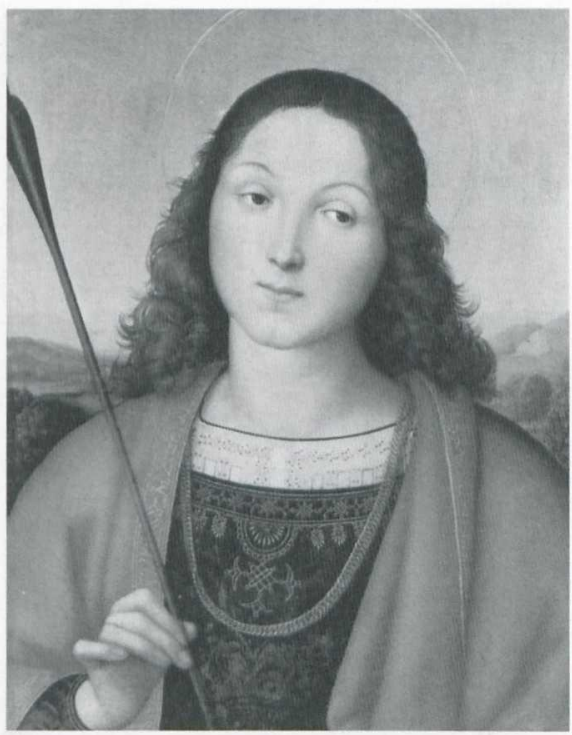

von Schmerz, Schönheit, Leid und eben Begehren verknüpft ist. Die Darstellung dient also nicht nur der Repräsentation und Kontemplation idealer Schönheit, sondern zielt auch und vor allem auf die subjektive und affektive Involvierung des - männlich zu denkenden - Betrachters vor dem Gemälde, der der verführerischen Macht des Knaben unterliegt.

Koos' Argumentation ist stringent, doch bleibt vor dem Gemälde und anderen vergleichbaren Knabenbildern mit Pfeil ein Moment der Irritation bestehen. Es ist die Folge der Verwendung eines fest kodifizierten Symbols, des Pfeils, bei gleichzeitiger Existenz sowohl dieser Bildform für Heiligendarstellungen - wie Raffaels Sebastian -, als auch der Existenz tendenziell ambiger Darstellungen, in denen sich, wie in Parmigianinos Barbara, profane und sakrale Elemente und Gestaltungsweisen wechselseitig überlagern. Ob Giorgione dieses Gefühl der Irritation des Betrachters möglicherweise einberechnete oder gar beabsichtigte, ist aus der historischen Distanz bei einer sehr geringen Zahl von Rezeptionsdokumenten sehr schwer einzuschätzen. ${ }^{23}$ Indirekt können hierauf aber die Existenz und vor allem die Rezeptionsgeschichte einiger zeitgleich entstandener lombardischer Knabenbilder in Halbfigur den Hinweis geben, die Giorgiones Knabe mit Pfeil in der Anlage sehr ähnlich sind. So gibt es von Giovanni Antonio Boltraffio, einem Maler aus dem Umkreis Leonardos, sowie von Marco D'Oggiono eine ganze Reihe von Darstellungen lieblicher und teilweise äußerst vornehm gekleideter und kostbar ge-

23 In diese Richtung denkt Christian Hornig, der davon ausgeht, Giorgione habe in dem Gemälde „spielerisch verschiedene Sinnschichten verzahnt“ (Hornig, a. O. Anm. 17, S. 212). 


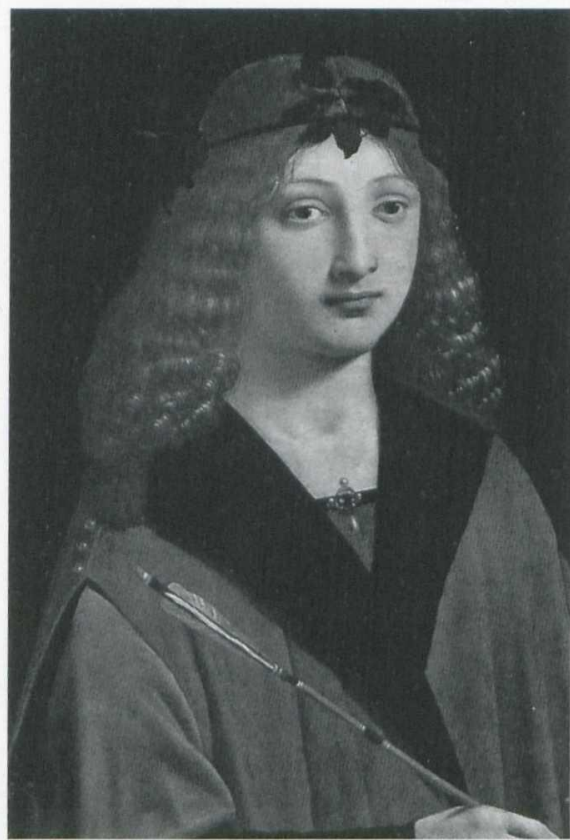

Abb. 4: Giovanni Antonio Boltraffio, Bildnis eines Knaben mit Pfeil; San Diego, Timken Museum of Art.

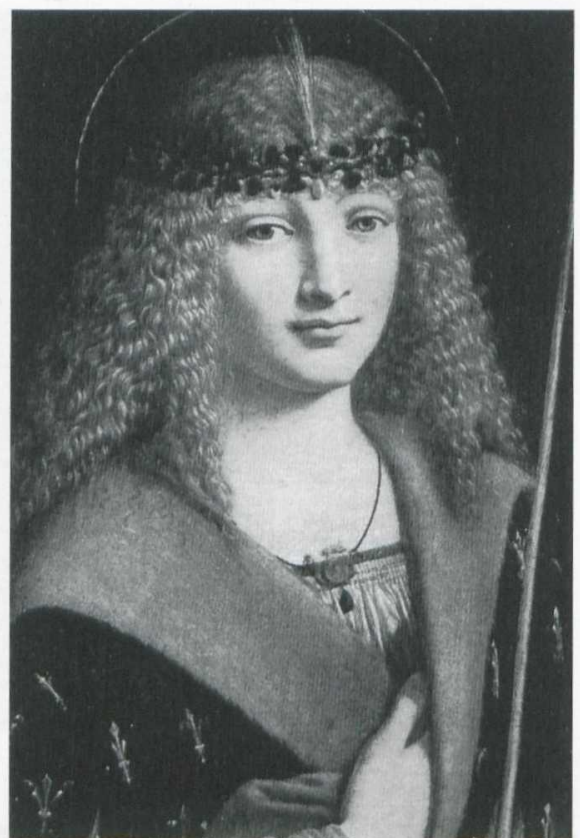

Abb. 5: Ders., Bildnis eines Knaben mit Pfeillhl. Sebastian; Moskau, PuschkinMuseum. (Farbtafel 22)

schmückter Knaben mit einem Pfeil in der Hand (Abb. 4-6). ${ }^{24}$ Interessanterweise verfügt ein Teil von ihnen über Heiligenscheine (Abb. 5+6), ${ }^{25}$ ohne dass die Darstellungsweise der Knaben in signifikanter Weise von denen ohne Nimben differierte. Unmissverständlich ist der Heiligenschein in Boltraffios Knabenbild der Sammlung Frizzoni (Abb. 6) inschriftlich als „S. Sebastianus“ bezeichnet. Wir wis-

24 So sein Gemälde im Timken Museum of Art in San Diego; siehe Maria Teresa Fiorio, Giovanni Anontio Boltraffio. Un pittore milanese nel lume di Leonardo, Mailand/Rom 2000, S. 94-96, Nr. A8 sowie die Werke in der folgenden Anmerkung. Für den Leonardo-Schüler Giovanni Antonio Boltraffio (*um 1467, Mailand, † 1516 ebd.), siehe ebd. und E. M. Dal Pozzolo, „Giovanni Antonio Boltraffio", in: Saur Allgemeines Künstlerlexikon, XII, München/Leipzig 1996, S. 425 f.; für Marco d'Oggiono, der ebenfalls der Werkstatt Leonardos angehörte und mit Boltraffio gemeinsam arbeitete: Janice Shell, „Marco d'Oggiono: Oggiono, c. 1475 - c. 1530“, in: The Legacy of Leonardo. Painters in Lombardy 1490-1530, mit Essays v. Giulio Bora u. a, Mailand 1998, S. 163-178.

25 Siehe hierfür Koos (2006), a. O. Anm. 17; für die Gemälde im Moskauer Puschkin Museum (Holz, auf Lw. übertragen; 46 x 34cm, ca. 1498/99) und ehem. in der Sammlung Frizzoni, Bergamo, später Sammlung Scaglioni Frizzoni, Messina (Holz, ohne Maßangaben; ca. 1498-90) siehe Fiorio, a. O. Anm. 24, S. 89 f., Nr. A6 und 212, E3; Elena Rama, „Un tentativo di rilettura della ritrattistica di Boltraffio fra Quatrocento e Cinquecento", in: Arte Lombarda 64 (1983), S. 79-92, hier S. 82. 84. Für das Gemälde von D’Oggioni siehe Rama (1983), S. 82, Abb. 9 (ohne Maßangabe). 
Abb. 6: Ders., hl. Sebastian; ehem. Sammlung Frizzoni.

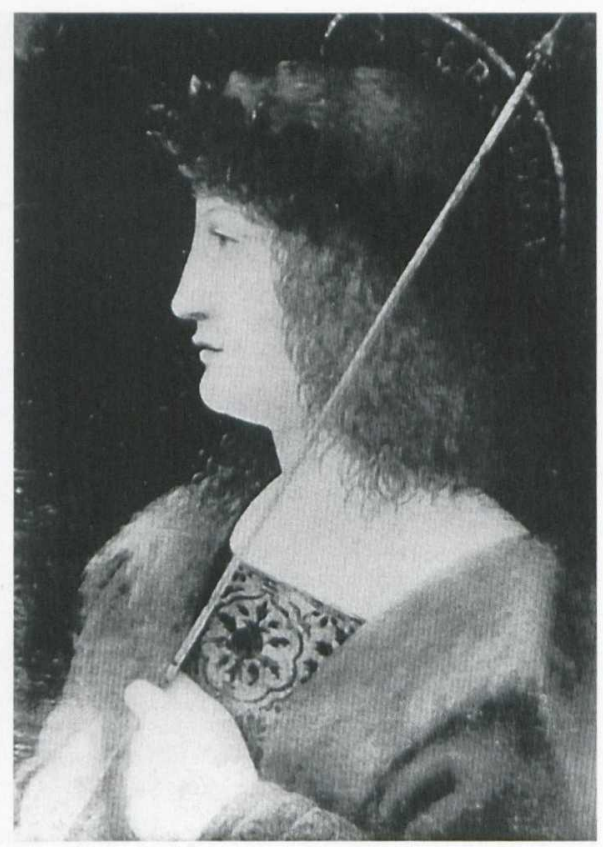

sen, dass im Moskauer Gemälde Boltraffios (Abb. 5) der Nimbus des Knaben nachträglich hinzugefügt wurde; wann dies allerdings geschehen ist, ist ebenso un$\mathrm{klar}^{26}$ wie die Bedeutung dieses Vorgangs, also ob eine von Boltraffio als „Sebastian" gemeinte Darstellung von anderer Hand (?) lediglich „vereindeutigt" wurde, oder ob bei diesem Eingriff das Sujet entsprechend verändert wurde. Für äußerst unwahrscheinlich halte ich es allerdings, dass alle genannten Figuren mit Nimbus erst nachträglich in einen "Sebastian" verwandelt wurden. ${ }^{27}$

Wie dem auch sei: Dass der Reiz dieser Knabenbilder wohl bereits für die Zeitgenossen auch und möglicherweise sogar gerade in der semantischen Offenheit der Darstellungen lag, dürfte durch diese Beispiele deutlich werden. Sie bot das Potential, die Darstellungen performativ zu transformieren: Von einem einfachen Knaben mit Pfeil, der die Themen der Schmerzliebe aufruft und das Begehren seiner (wohl überwiegend) männlichen Betrachter schürt, in liebliche Darstellungen des hl. Sebastian bzw. entsprechende Rollenporträts, die diese Effekte kaum weniger erzeugen und gerade darin den profanen androgynen Knaben vergleichbar sind. Der Reiz für die Betrachter hat in meinen Augen weniger im Sinne Salvatore Settis

26 Fiorio, a. O. Anm. 24, S. 90.

27 Anders Koos (2006), a. O. Anm. 17, S. 123, die davon ausgeht, in allen Knabenbildern seien die Nimben , in Unkenntnis oder aber zur Sublimierung des Bildgegenstands“ ergänzt worden. Ich kann mir kaum vorstellen, dass späteren Rezipienten die Existenz einer Bildgattung nicht mehr bekannt gewesen sein soll. 
im „Rätsel-Raten“ über das Sujet der Bilder, ${ }^{28}$ als vielmehr im Nachvollzug dieses performativen Wandlungsprozesses und in der Spannung zwischen einem sakralen und einem profanen Sujet bestanden. Dieser Nachvollzug des Potentials der Bilder wird einhergegangen sein mit der Wahrnehmung der gezielten Differenz der Figuren zur traditionellen Darstellungsweise und Ikonographie des hl. Sebastian, wie sie insbesondere in Boltraffios Moskauer Knaben mit seinem perlenbesetzten Diadem mit Feder und der Hand im Mantelaufschlag gegeben ist. ${ }^{29}$ Aber nicht nur im Rezeptionsvorgang wird dieses Faszinosum bestanden haben, sondern auch im produktiven Akt der „Verwandlung“ der Knaben selber, ihrer Überführung von der profanen in die sakrale Ebene und dem Ausreizen des ihnen eigenen Ambiguitätspotentials.

\section{Magdalena}

Dass sich dies keineswegs auf Knabenbilder beschränkte, zeigt eine Vielzahl von Magdalena-Darstellungen - eine Heilige, die sich aufgrund ihrer Lebensgeschichte und heilsgeschichtlichen Bedeutung als die große Konvertin des Neuen Testamentes, von der Lukas berichtet, dass sie „viel geliebt“ und gesündigt habe (Lk 7, 47), für solche ambigen, zwischen profanen und sakralen Gestaltungsweisen oszillierenden Darstellungen in besonderer Weise eignete. Wie bewusst dieses ambivalente Potential der Figur in der Frühen Neuzeit ausgelotet wurde, können Giovanni Gerolamo Savoldos Darstellungen der Heiligen in Halbfigur deutlich machen. Was wir an Boltraffios Knabenbildern beobachten konnten, nämlich ein Interesse am Verwandeln der Figur, das sich ihr genuines Ambiguitätspotential zunutze macht, begegnet uns auch bei Savoldo, und hier können wir durch eine bessere Dokumentation der Bilder die Absichten des Malers sicherer rekonstruieren.

Es handelt sich insgesamt um vier als original geltende und voneinander abhängige Gemälde, die vermutlich in den ausgehenden zwanziger und frühen dreißiger Jahren des 16. Jahrhunderts entstanden sind und sehr erfolgreich waren, nimmt man als Indikator hierfür die Nachstiche und überhaupt das Faktum der Vielzahl von Bildfassungen. ${ }^{30}$ Als Prototyp wird meist das Gemälde in der Londoner Nati-

28 Koos (2006), a. O. Anm. 17, S. 118 erwähnt, Settis habe in einem im Juli 2004 im Wiener Kunsthistorischen Museum gehaltenen Vortrag seine an Giorgiones Tempesta entwickelte Interpretationsidee für den Knaben mit Pfeil weiterentwickelt (Salvatore Settis, Giorgiones, Gewitter. Auftraggeber und verborgenes Sujet eines Bildes in der Renaissance, Berlin 1982).

29 Es handelt sich wohl um einen höfischen Gestus. Hinzuweisen ist darauf, dass der recht lange und vom Bildrand überschnittene Stab in der Hand des Knaben im Puschkin-Museum nicht zweifelsfrei als Pfeil zu lesen ist.

$30 \mathrm{Zu}$ den Gemälden: Renata Stradiotti, in: Sybille Ebert-Schifferer (Hrsg.), Giovanni Gerolamo Savoldo und die Renaissance zwischen Lombardei und Venetien. Von Foppa und Giorgione bis Caravaggio (Ausst.-Kat. Frankfurt/Brescia 1990), Mailand 1990, S. 140-147. Als original gelten inzwischen folgende vier Fassungen:

1.) London, National Gallery; 89,1 x 82,4 cm; Stradiotti (1990), S. 140-143, Nr. I 13 (Dat.: ca. 1528-1530); Nicholas Penny, National Gallery Catalogues. The Sixteenth Century Italian 
Abb. 7: Giovanni Gerolamo Savoldo, hl. Maria Magdalena; London, National Gallery. (Farbtafel 23)

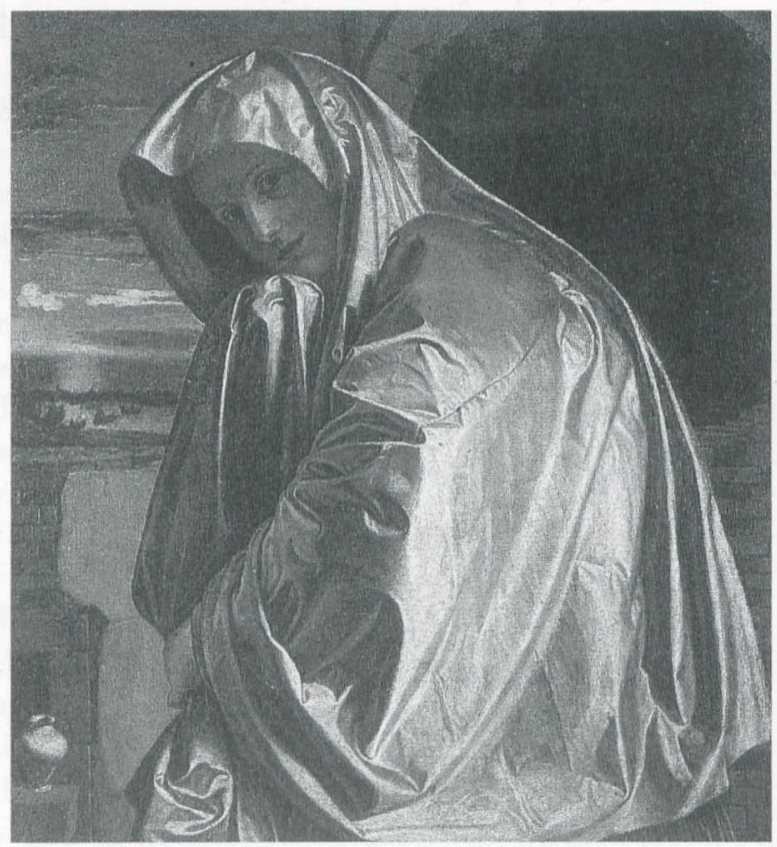

onal Gallery (Abb. 7) mit den Maßen $84 \times 79 \mathrm{~cm}$ angesehen. ${ }^{31}$ Es zeigt in Halbfigur eine junge Frau ohne Nimbus, die in einen großen silbrig glänzenden Umhang gehüllt ist, der auch ihr Haar und die rechte Hand dezent bedeckt. Sie steht vor einer ruinösen Architekturkulisse mit einer Arkade, hinter der ein verfallenes Haus mit Portiken und schmalen Rundbogenfenstern zu erkennen sind. Die niedrige, bildparallel geführte Mauer auf der linken Seite ermöglicht den Ausblick in die ferne Landschaft, in der ein Turm, Wasser und Boote erkennbar sind. Die besondere Ausleuchtung lässt auf einen Sonnenauf- oder Sonnenuntergang schließen,

Paintings. I Paintings from Bergamo, Brescia and Cremona, London 2004, S. 346-353, Nr. 1031 (Dat.: 1535-40); Cecil Gould, National Gallery Catalogues. The Sixteenth-Century Italian Schools, London 1975, S. 236 f. Nr. 1031.

2.) Berlin, Gemäldegalerie SMPK; 94,1 x 75,3 cm; ca. 1528-1531; Stradiotti (1990), S. 144 f. Nr. I 14. Staatliche Museen Preußischer Kulturbesitz, Berlin, Gemäldegalerie. Katalog der ausgestellten Werke des 13.-18. Jahrhunderts, Berlin 1975, S. 391, Nr. 307 (Die Venezianerin).

3.) Los Angeles, J. Paul Getty Museum (ehem. Zürich Privatsammlung/Warwick Castle); $99 \mathrm{x}$ $80 \mathrm{~cm}$; 1530er Jahre; Stradiotti (1990) S. 146 f. Nr. I 15.

4.) Florenz, Sammlung Contini Bonacossi, ausgestellt in der Galleria Palatina/Palazzo Pitti, $84 \mathrm{x}$ 77,5 cm; Gli Uffizi. Catalogo Generale, Florenz 1979, S. 472, Nr. P1423.

Die Datierung der verschiedenen Fassungen schwankt in der Forschung stark; vgl. auch Creighton Gilbert, The Works of G. Savoldo, Diss. 1955, S. 164.172 und 176 sowie ders., The Works of Girolamo Savoldo. The 1955 Dissertation with a Review of Research 1955-1985, New York/London 1986, S. 555 f.; Schleier (1991). Ich folge Stradiotti und Schleier (Anm. 39) in der oben angegebenen Reihenfolge.

31 Für das Gemälde siehe die vorherige Anm., Nr. 1. 
wobei die Lichtquelle nicht identisch sein kann mit derjenigen, welche die Figur ausleuchtet, denn diese ist vor dem Bild zu denken.

Die Haltung der Figur und ihre Anordnung im Bildfeld sind ungewöhnlich: mit dem Körper ist sie uns von der Seite zugewandt - wobei ihr linker Ellenbogen die ästhetische Oberfläche zu verletzen scheint -, den Kopf dreht sie uns leicht gesenkt und wohl gestützt auf die in das Tuch gewickelte rechte Hand zu, so als suchte sie den Blickkontakt zum externen Betrachter.

Die moderne Ikonologie hätte die Benennung dieses Sujets, für das in dieser Form keine Bildtradition existiert, sicherlich vor größere Rätsel gestellt, ließe sich das Gemälde nicht mit Ottavio Rossis secentesker Erwähnung „una bellissima Maddalena coperta da un pan bianco “32, einer „sehr schönen, in ein weißes Tuch gehüllten Magdalena" in Verbindung bringen. Auf diese Bezeichnung der Figur als Magdalena verweist auch das kleine Salbgefäß in der linken unteren Bildecke, das dort auf einem Stein abgestellt ist. ${ }^{33}$ Mary Pardo hat in einem Aufsatz von 1989 vorgeschlagen, das Gemälde auf die von Joh. 20, 11-18 geschilderten Ereignisse im Garten Gethsemane am Ostermorgen zu beziehen: ${ }^{34}$ Der Evangelist schildert, wie Magdalena weinend vor dem leeren Grab Christi steht, als ihr der Auferstandene erscheint. Sie hält ihn zunächst für den Gärtner, bis er sie mit ihrem Namen „Maria“ anspricht. Er prophezeit ihr seine Himmelfahrt mit dem Auftrag, davon den Aposteln Bericht zu erstatten. Nach Pardo zeigt Savoldo die hl. Magdalena im Akt der Konversion von der metaphorischen Blindheit zur Erkennenden - ein mentaler Vorgang, den ihre nach vorn gebeugte, sinnierende Haltung nachvollzieht. ${ }^{35}$ Trifft diese Lesart zu, besteht der Kunstgriff des Bildes darin, dass die Erscheinung des Herrn, dessen Position etwa am Standort des externen Betrachters zu denken ist, indirekt für uns sichtbar wird, weil er uns über Magdalenas Blick

32 Ottavio Rossi, Elogi historici di bresciani illustri, Brescia 1620, S. 520. Das Bild wird im Haus des Dott. Lorenzo Averoldo genannt. Ridolfi wird sich später darauf beziehen, die Attribute und auch die Existenz der vielen Kopien vermerken: Carlo Ridolfi, Le Maraviglie dell'arte ouuero le uite degli illustri pittori ueneti e delo stato, Venedig 1648, hrsg. \& komm. v. Detlev Frhr. v. Hadeln, Berlin 1914, I S. 271: „[...] in casa Auerolda in vna figura della Maddalena inuolta in drappo, col vase dell' alabastro, incaminata al Sepolcro, celebre pittura, della quale si sono tratte molte copie“. Diese Beschreibung wird aufgrund der Bezeichnung des Tuchs als „bianco“ auf die Londoner Fassung bezogen.

33 Zur Ikonographie der Figur und Bildtradition siehe Ingrid Maisch, Maria Magdalena, Freiburg u. a. 1996; Susan Haskins, Mary Magdalen, Myth and Metaphor, London 1993; Monika IngenhoffDanhäuser, Maria Magdalena. Heilige und Sünderin in der italienischen Renaissance. Studien zur Ikonographie der Heiligen von Leonardo bis Tizian, Tübingen 1984; Marilena Mosco (Hrsg.), La Maddalena tra sacro e profano. Da Giotto a De Chirico (Ausst.-Kat. Florenz), Florenz 1986.

34 Mary Pardo, „The Subject of Savoldo's Magdalene“, in: The Art Bulletin 71 (1989), S. 67-91; Gilbert (1955) und Gould (1975), a. O. Anm. 30, bezogen die Darstellung allgemeiner auf Johannes' Schilderung von Magdalenas Gang zum Grab Christi (Joh. 20,1).

35 Pardo, a. O. Anm. 34, S. 74: „To use the language of the Vulgata (conversa est retrorsum, conversa illa), the Magdalen depicted by Savoldo is experiencing an aspect of conversion both literally and on a plane that redefines (and fulfilles) the very modality of her role as Christian example: repentant sinner, convert." 
Abb. 8: Ders., hl. Maria

Magdalena; Los Angeles,

J. Paul Getty Museum.

(Farbtafel 24)

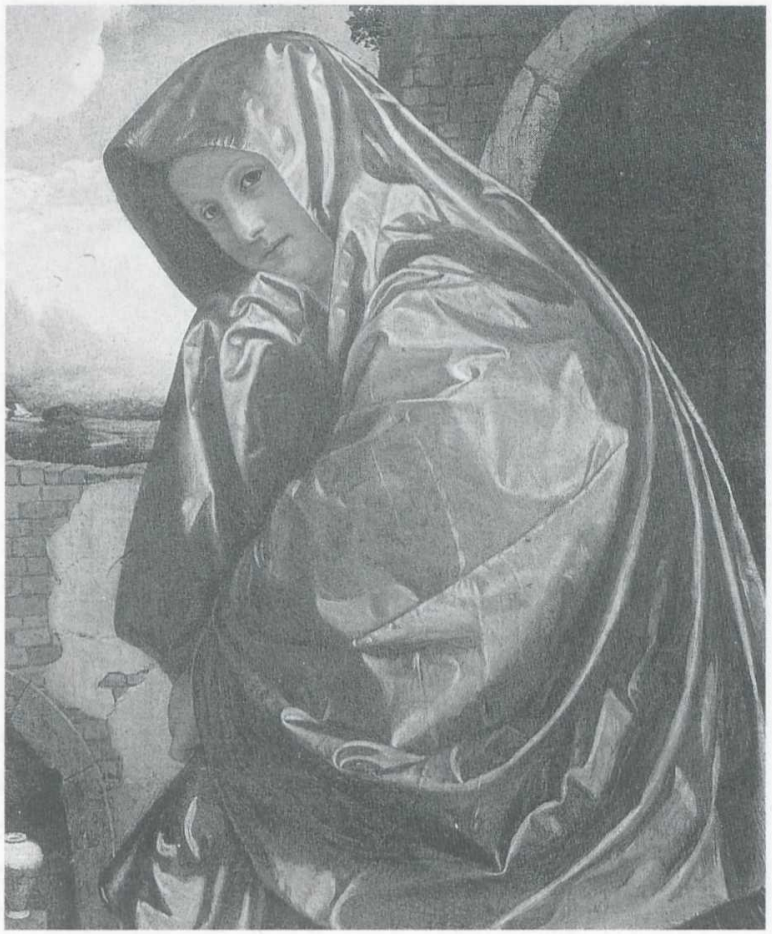

und den Lichtglanz ihres Tuchs vermittelt wird. ${ }^{36}$ Dass dieses Wissen um die Nähe des Herrn und seine Worte für Magdalena tröstlich sind, darauf verweist die Gebärde der rechten Hand, mit der sie sich die Tränen zu trocknen scheint.

So nachvollziehbar diese Deutung von Savoldos Gemälde auch ist, so gilt es nichtsdestotrotz die Ungewöhnlichkeit dieses Bildentwurfs zu betonen, die ja insbesondere für die Rezeption des Werks Folgen hat. Wir haben keine Darstellung der Erscheinung des auferstandenen Jesus Christus vor Magdalena vor uns, wie sie traditionell im ikonographischen Schema des „Noli me tangere“ visualisiert wurde. Savoldo verzichtet auch auf konventionalisierte Zeichen einer Erleuchtung der Heiligen etwa mittels verzückter Augen oder eines himmelwärts gerichteten Blicks, und es fehlen alle weiteren Figuren oder Attribute wie die Engel am leeren Grab oder der Berg Golgatha mit den drei Kreuzen im Hintergrund, welche uns und den zeitgenössischen Betrachtern die Identifizierung dieses ungewöhnlichen Sujets erleichterten, im Gegenteil: Wir können zwar erahnen, dass sich in der Nische ein

36 Siehe hierzu Klaus Krüger, Das Bild als Schleier des Unsichtbaren. Ästhetische Illusion in der Kunst der frühen Neuzeit in Italien, München 2001, S. 104-106: „Eben diese Grenzerfahrung menschlicher Sehens- und Erkenntniskraft übermittelt das Gemälde auch dem externen Betrachter, dergestalt $\mathrm{da} ß$ sich ihm die unsichtbare - und folglich auch nicht abbildbare - Göttlichkeit des Auferstandenen allein im so lebendig schimmernden Widerspiel des Lichtes auf dem Tuch offenbart, in welches die Heilige sich hüllt" (S. 105). 


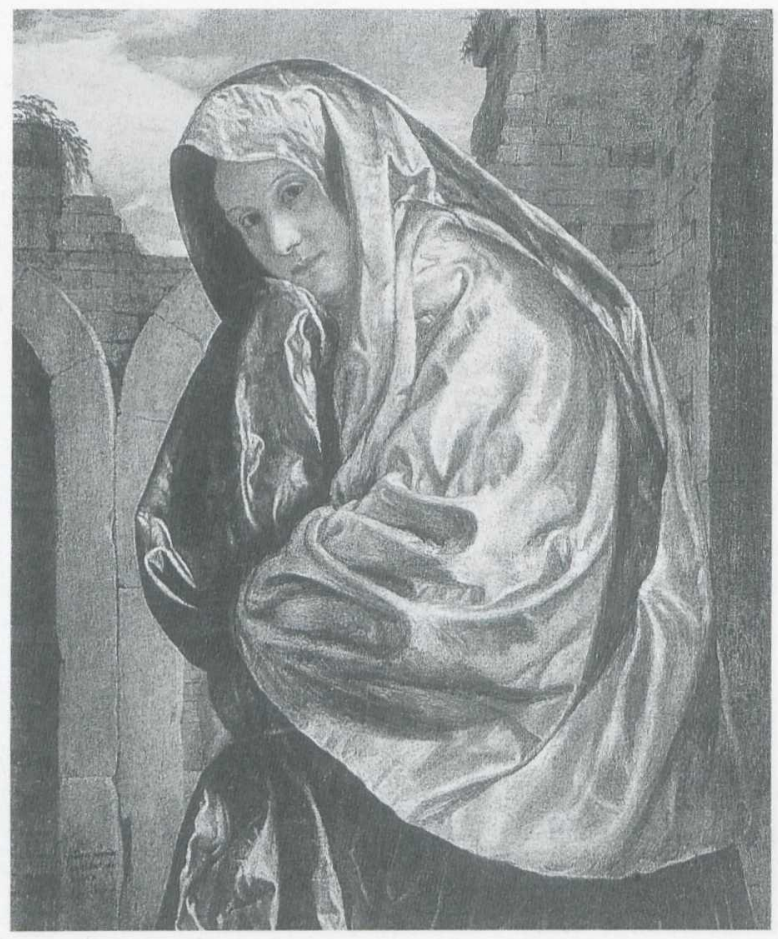

Abb. 9: Ders.,

„La Veneziana“; Berlin, Gemäldegalerie SMPK. (Farbtafel 25)

Grab verbirgt; dass es aber leer ist - was ja Fokus der Darstellung sein müsste -, wird uns nicht gezeigt, und auch die venezianische Lagunenlandschaft lenkt die Wahrnehmung des Bildes kaum in die Richtung, hier den Garten Gethsemane zu vermuten.

Nicht nur, dass bereits diese Bildformulierung das Thema der verweisenden Kraft von Bildzeichen und der Darstellungsweise aufwirft, Savoldo variiert sie auch in signifikanter Weise, was in meinen Augen seine Reflexion über das hier interessierende Thema der Generierung von „Inhalt" und „Bedeutung" deutlich werden lässt. ${ }^{37}$ So ist in dem Gemälde im Getty Museum (Abb. 8), ${ }^{38}$ in der Magdalena nun einen goldenen Mantel trägt, zwar die im Londoner Gemälde irritierende Seelandschaft verändert, allerdings passen auch die Rundhäuser mit Spitzdächern, hinter denen ein Gebirgszug verläuft, nicht zum biblischen Bericht, und es fehlt zugleich jeder Hinweis auf einen Sonnenaufgang, der neben dem - auch hier wiederum nicht als leer gekennzeichneten - Grab ja eine Voraussetzung für die Bestimmbarkeit und natürlich auch den Sinn des Sujets bildet.

37 Die Reihenfolge der Entstehung der Bilder ist nicht gesichert; ich halte mit Stradiotti, a. O. Anm. 30, und Schleier, a. O. Anm. 39, die in Anm. 24 angegebene für die wahrscheinlichste.

38 Siehe oben, Anm. 24., Nr. 3. 
Abb. 10: Correggio, Liegende Maria Magdalena (zerstört); ehem. Dresden, Gemäldegalerie. (Farbtafel 26)

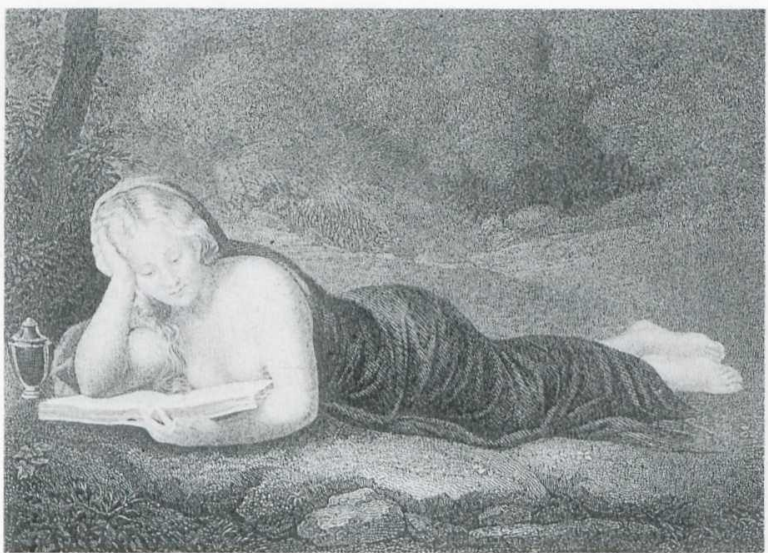

Noch viel gravierender allerdings sind die Veränderungen auf dem Gemälde in der Berliner Gemäldegalerie (Abb. 9), ${ }^{39}$ das den Ausschnitt zwar leicht vergrößert, dabei aber auf alle die Benennung der Figur und des Sujets leistenden Attribute verzichtet: das Salbgefäß, das Grab und die Landschaft mit Sonnenaufgang. Auch die Gestaltung von Magdalenas wiederum goldenem Mantel ist durch die stärkere und unruhige Faltenbildung verändert, was die postulierte Bildidee einer Reflexion des Lichtscheins Christi durch den Mantel unterläuft. Dass es sich bei dem Berliner Gemälde nicht um eine derivate, einem „unverständigen“ Werkstattangehörigen zuschreibbare Fassung handeln kann, bezeugt neben der Qualität der Ausführung ${ }^{40}$ der Umstand, dass ausgerechnet dieses Gemäldes ausführlich mit „JOANES JERONIUS SAUOLDUD. D. BRISIA FACIEBAT" signiert ist. Bedauerlicherweise können wir die Provenienz dieses Bildes nur bis ins 19. Jahrhundert zurückverfolgen und wissen folglich nicht, wie die Zeitgenossen es beschrieben: La Veneziana ist der heute gängige, unspezifische Bildtitel, ${ }^{41}$ und Monika Ingenhoff-Danhäuser erkennt in der weiblichen Figur sogar eine Kurtisane, weil das über die Stirn gezogene Tuch der Dame Merkmal der Tracht einer venezianischen Kurtisane war. ${ }^{42}$ Die sich an diesem Beispiel manifestierenden Ambivalenzen sind, wie gese-

39 Siehe oben, Anm. 24, Nr. 2; sowie Erich Schleier, "Savoldos Magdalena in der Berliner Gemäldegalerie. Zur Wiederherstellung eines Meisterwerks der Brescianer Cinquecentomalerei“, in: Jahrbuch der Berliner Museen 33 (1991), S. 135-147.

40 Ebd., S. $144 \mathrm{f}$.

41 Vgl. Stradiotti, a. O. Anm. 30, S. 144; so etwa Tamara Fomiciova, „Giorgione e la formazione della pittura di genere nell'arte veneziana del XVI secolo", in: Giorgione. Atti del convegno Internazionale di Studio per il $5^{\circ}$ Centenario della nascita (Castelfranco Veneto 1978), Venedig 1979, S. 159-164, hier S. 163.

42 Ingenhoff-Danhäuser, a. O. Anm. 33, S. 59-61. Die Tracht verzeichnet Cesare Vecellio, Habiti antichi et moderni di tutto il mondo, Venedig 1598, S. 107: Cortigiane fuor di casa: „Quelle meretrici, che vogliono acquistar credito col mezo della finta honestà, si seruono dell'habito uedouile, \& di usanza nô ancora dismeßa affatto, bêche usata con modestia maggiore. di maniera che non potendo star sempre serrate, \& coperte cô la cappa, che portano, \& non potendo d'altra parte esser 


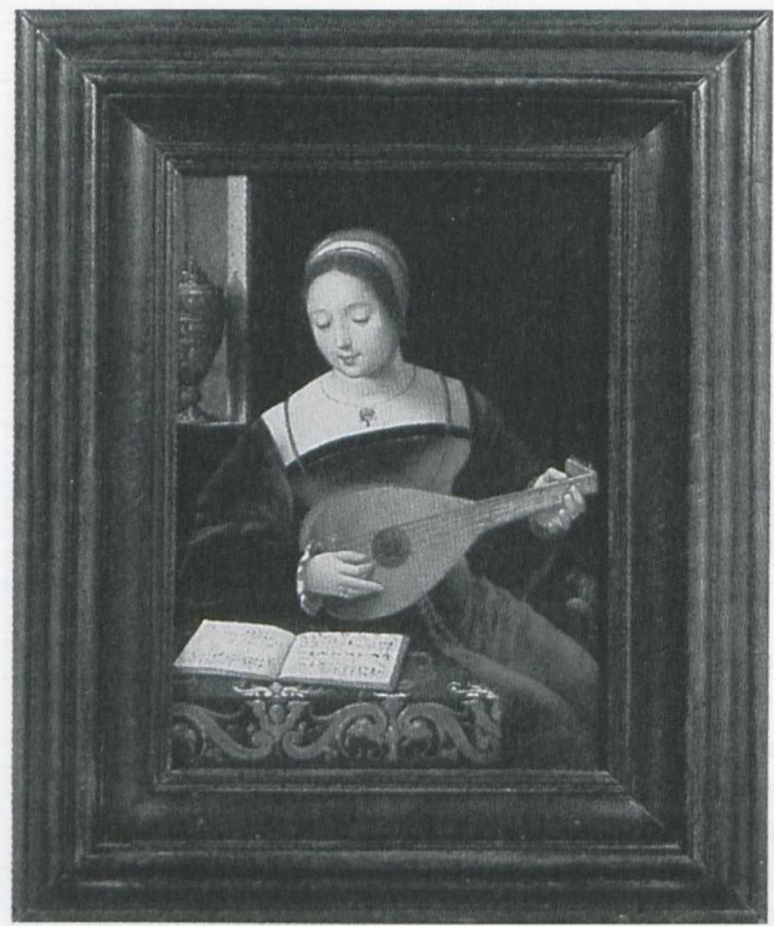

Abb. 11: Meister der weiblichen Halbfiguren, Hofdame beim Lautenspiel; Hamburg, Kunsthalle.

hen, keineswegs beispiellos in der Malerei des Primo Cinquecento; die Besonderheit besteht hier darin, dass wir durch die Vielzahl signifikant variierender Bildfassungen die Genese, und damit den "visuellen“ Denkprozess des Künstlers so gut verfolgen können.

In diesem Kontext lassen sich eine ganze Reihe von Magdalena-Gemälden mit ähnlich ambigem Charakter anführen, zu denen es bezeichnenderweise bewegte Forschungsdiskussionen bezüglich ihrer adäquaten Benennung und Zuordenbarkeit zu bestimmten Gattungen gibt. Ich nenne Correggios kleinformatige auf einer Kupferplatte ausgeführte, liegende und in einem großen Buch lesende Magda-

uedute, sono finalmête sforzate scoprirsi alquanto, \& è perciò iompossibile, ch'elle non sieno conosciute à qualche gesto. \& perche sono loro prohibite le perle, sono in particolare conosciute per tali, quâdo mostrano scoperto il collo, vestono del resto pomposamente: sotto usano brocadelli di seta, come anco calze ricamate, cosi carpette, \& camicie." Analog vermutet Ingenhoff-Danhäuser in den Variationen des Gemäldes, in denen ein Salbgefäß auf die hl. Magdalena verweist, ein Rollenbildnis, also eine Kurtisanenporträt in Gestalt Magdalenas, womit „Heiligen- und Kurtisanenikonographie in einem Bild vereinigt sind“ (S. 60). Ihre auf Seite 70 angestellten Überlegungen bezüglich einer moralisierenden Komponente der Darstellungen im Zuge erster gegenreformatorischer Tendenzen in Venedig kann ich nicht nachvollziehen, zumal die Autorin die Werke dadurch in die vierziger Jahre zu datieren geneigt ist, was nicht überzeugt. Kritisch hierzu auch Pardo, a. O. Anm. 34, S. 71, Anm. 10. 
Abb. 12: Giampetrino, Egeria (?); Mailand, Sammlung Brivio Sforza. (Farbtafel 27)

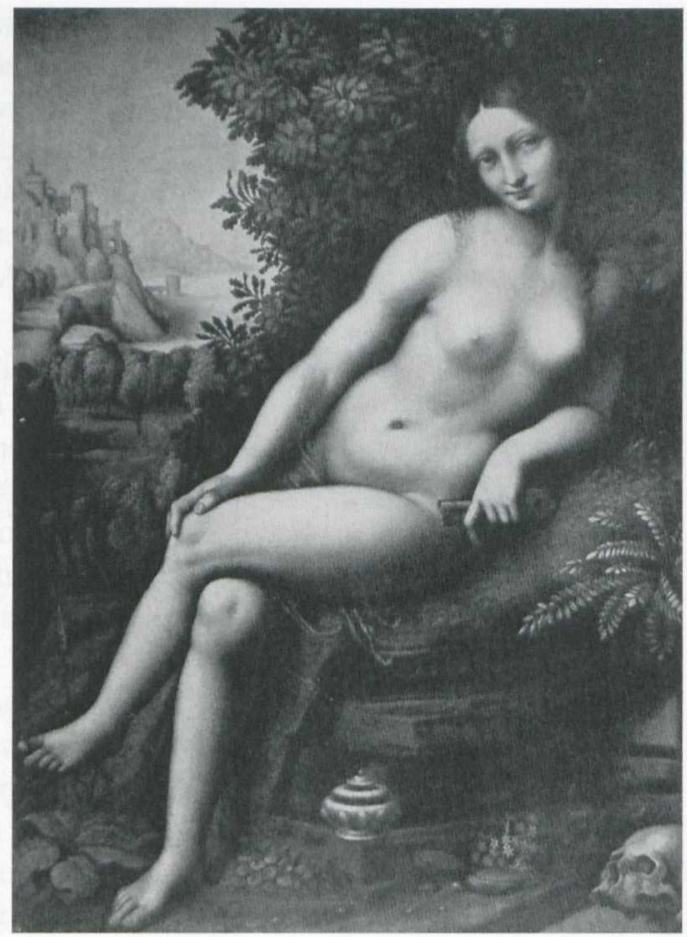

lena, die sich ehemals in der Dresdner Gemäldegalerie befand (Abb. 10). ${ }^{43}$ Sie ist allein durch das neben ihr stehende Salbgefäß als hl. Magdalena ausgewiesen. Die genrehafte Gestaltung mit ihr dem auf den rechten Arm gestützten Kopf in Verbindung mit dem weiten Dekolleté der Figur ist besonders reizvoll, was den sich wiederum in zahlreichen Reproduktionen manifestierenden großen Erfolg des Bildes erklärt. Von der bekennenden und einsam in einer Höhle büßenden Sünderin ist eine solche Darstellung weit entfernt. Ob die in der Hamburger Kunsthalle aufbewahrte Hofdame beim Lautenspiel eines flämischen, in Antwerpen tätigen Meisters (Abb. 11) durch das große Salbgefäß hinter ihr auf dem Fensterbrett ebenfalls als Magdalena oder als entsprechendes Rollenporträt einer Dame bezeichnet ist, ist in der Forschung ebenso umstritten ${ }^{44}$ wie die Identität der weiblichen Aktfigur vor einer Felsgrotte in dem Gemälde des Giampetrino in der Mai-

$4329 \times 39,5$ cm; Kriegsverlust; zu diesem Gemälde und seinem Rezeptionserfolg jüngst: Maddalena Spagnolo, „Correggio's Reclining Magdalen. Isabella d'Este and the Cult of St Mary Magdalen“, in: Apollo 157 (2003), Nr. 496, S. 37-45.

44 Siehe für das Gemälde Martina Sitt, „Meister der weiblichen Halbfiguren“, in: Alte Rahmen - neue Sicht (=Hefte der Hamburger Kunsthalle), Hamburg 2000, o. Pag. Für weitere Informationen danke ich der Autorin. 
länder Sammlung Brivio Sforza (Abb. 12), ${ }^{45}$ deren Symbole - das ostentativ zur Schau gestellte Salbgefäß und der Totenschädel - ebenso wie die Einbindung der Figur in die Landschaft auf eine Magdalena hinweisen. Gleichwohl bleibt die Darstellungsweise einer emotional unbewegten und vor allem gänzlich nackten Figur, die ihren Blick auf den Betrachter richtet, irritierend, weshalb die Figur überwiegend als die italische Quellnymphe Egeria, Gattin oder Geliebte des römischen Königs Numa Pompilius, identifiziert wird. ${ }^{46}$ Allerdings fehlt jeder innerbildliche Hinweis auf eine Quelle, ${ }^{47}$ und darüber hinaus passen zu dieser ikonographischen Bestimmung weder die Attribute des Salbgefäßes und Totenschädels, ${ }^{48}$ noch erklärt sich der Schlüssel in der Hand der Dame. Er gehört allerdings auch nicht zur Magdalenen-Ikonographie. Da eine „interpretatio Christiana“ der heidnischen Figur angesichts der Einmaligkeit des Belegs wohl ausscheidet und in den bekannten Beispielen für solche religiösen Umdeutungen auch durchgängig gerade die Attribute die Zugehörigkeit der Darstellung zu einem bestimmten Themenkreis bzw. generell der sakralen Bildwelt deutlich machen, ${ }^{49}$ ist hier von einer gezielten Uneindeutigkeit auszugehen.

Giampetrino war Schüler Leonardos. ${ }^{50}$ Diesem ist bekanntlich eine besondere Neigung zu intendiert ambigen Gemälden zu attestieren; ich verweise nur auf die Darstellung Johannes des Täufers in Halbfigur und des sogenannten JohannesBacchus im Louvre. ${ }^{51}$ Aber auch die Invention einer Magdalena in Halbfigur mit einem großen Salbgefäß in der Hand scheint nach Ausweis einer Zeichnung mit zwei Figurenskizzen in der Sammlung des Courtauld Institute auf ihn zurückzu-

45 Siehe Pietro C. Marani, „Giovan Pietro Rizzoli, called Giampetrino: Milan, documented from c. 1495 to 1549 “, in: The Legacy, a. O. Anm. 24, S. 275-300, hier S. 279 (um 1505-10); IngenhoffDanhäuser, a. O. Anm. 33, S. 25-27, 60, Wilhelm Suida, Leonardo und sein Kreis, München 1929, S. 214, 300. Das Gemälde ist auf der Felskante unterhalb des Salbgefäßes als „Egeria“ bezeichnet; $\mathrm{m}$. W. gibt es keine technischen Untersuchungen, die die Originalität dieser Inschrift untersucht hätten.

46 Piero Chiara/Gian Alberto Dell'Acqua u. a. (Hrsg.), Sacro e profano nella pittura di Bernardino Luini (Ausst.-Kat. Varese 1975) Cinisello Balsamo/Mailand 1975, S. 61; Suida, a. O. Anm. 45, S. 300. Für nicht überzeugend halte ich die von Ingenhoff-Danhäuser, a. O. Anm. 33, S. 60 aufgegriffene Deutung, es handele sich um ein (Rollen-)Porträt der Maitresse von Charles Amboise, dem französischen Gouverneur von Mailand, da die Gesichtszüge der Figur eindeutig der leonardesken Idealphysiognomie folgen; bezeichnenderweise sind sie mit denen von Giampetrinos Leda in der Kasseler Gemäldegalerie identisch.

47 Es sei denn, man liest das Gefäß als Verweis auf eine Quelle.

48 Siehe Der Neue Pauly. Enzyklopädie der Antike, hrsg. v. Hubert Cancik, Helmuth Schneider, Stuttgart 1997, III, S. 888; W. H. Roscher (Hrsg.), Ausfiibrliches Lexikon der griechischen und römischen Mythologie, Leipzig 1884-86, I, S. 1216 f.

49 Vgl. Ingenhoff-Danhäuser, a. O. Anm. 33, S. 26 f.

$50 \mathrm{Zu}$ Identität und Werk des Malers, der mittlerweile als identisch mit Giovanni Pietro Rizzoli gilt, siehe Marani, a. O. Anm. 45, S. 275-300; Francesco Frangi, „Giampetrino“, in: Mina Gregori (Hrsg.), Pittura a Milano. Rinascimento e Manierismo, Mailand 1998, S. 243-245; Janice Shell/ Grazioso Sironi, „Some documents for Giovanni Pietro Rizzoli: il Giampetrino“, in: Raccolta Vinciana 25 (1993), S. 121-135.

51 Hierfür, vor allem unter dem Gesichtspunkt der Ambiguität, jüngst Daniel Arasse, Leonardo da Vinci, Köln 1999, S. 461-473. 
Abb. 13: Leonardo, Studien für eine Magdalena; London, Courtauld Institute.

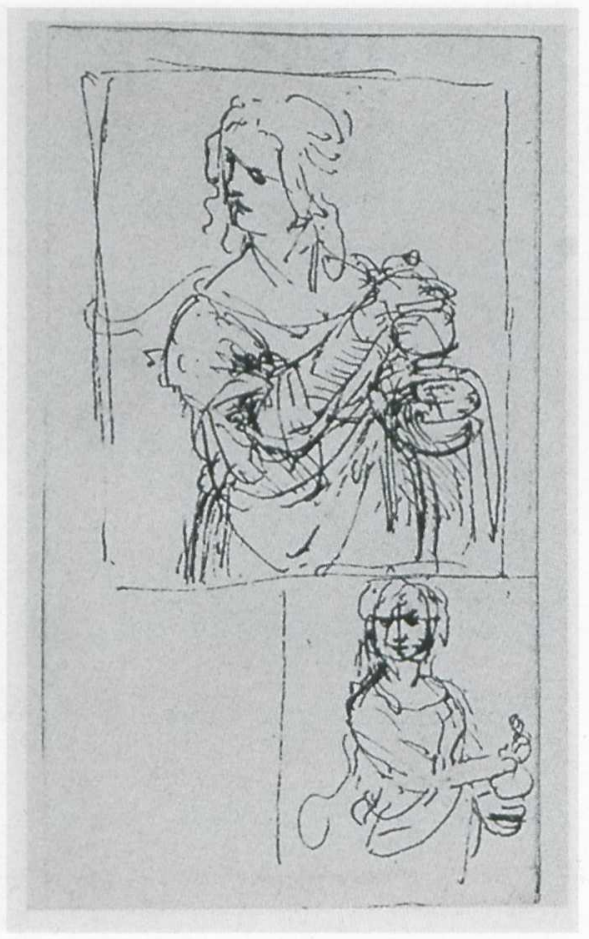

gehen (Abb. 13). ${ }^{52}$ Das Magdalena-Gemälde eines anderen Leonardo-Schülers, Bernardino Luini, das sich in der Washingtoner National Gallery befindet (Abb. 14), steht mit ihm in Verbindung. ${ }^{53}$ Anders als es dem ikonographischen Standard entspricht, zeigt es uns die Heilige nicht büßend, mit entblößtem Oberkörper und offenen, meist die Brust bedeckenden Haaren und gen Himmel gerichtetem Blick, sondern sorgsam gekleidet und frisiert, wobei sie den Betrachter konzentriert, aber ohne jede Emotion anblickt. Es ist neben den idealen Gesichtszügen allein das Attribut, die Schale, die sie öffnet und so innerbildlich aufwertet, das eine solche Darstellung von einem Porträt unterscheidbar macht. Gleichwohl liegt hier die Voraussetzung für die ambivalente Lektüre solcher Bilder, die dann

$5213,7 \times 7,9 \mathrm{~cm}$, Bleistift + Tinte, ca. 1480. Ehem. Princes Gate Collection; Carlo Pedretti, Leonardo. A Study in Chronology and Style, London 1973, S. 104; Antoine Seilern, Italian Paintings and Drawings at 56 Princes Gate London SW7, London 1959, S. 23, Nr. 80, und P1, XXXIV; Ingenhoff-Danhäuser, a. O. Anm. 33, S. 17; Pardo, a. O. Anm. 34, S. 80.

53 Holz; 59 × $84 \mathrm{~cm}$; Zu dem Gemälde von Luini siehe Pardo, a. O. Anm. 34, S. 81, IngenhoffDanhäuser, a. O. Anm. 33, S. 17; Angela Ottino Della Chiesa, Bernardino Luini, Novara 1956, S. 124, Nr. 193. Von Giampetrino gibt es eine ähnliche Darstellung in der Florentiner Sammlung Contini-Bonacossi (siehe Ingenhoff-Danhäuser, a. O. Anm. 33, S. 17 und Abb. 17), was die Abhängigkeit beider Gemälde von der erwähnten Zeichnung Leonardos sehr wahrscheinlich macht. 


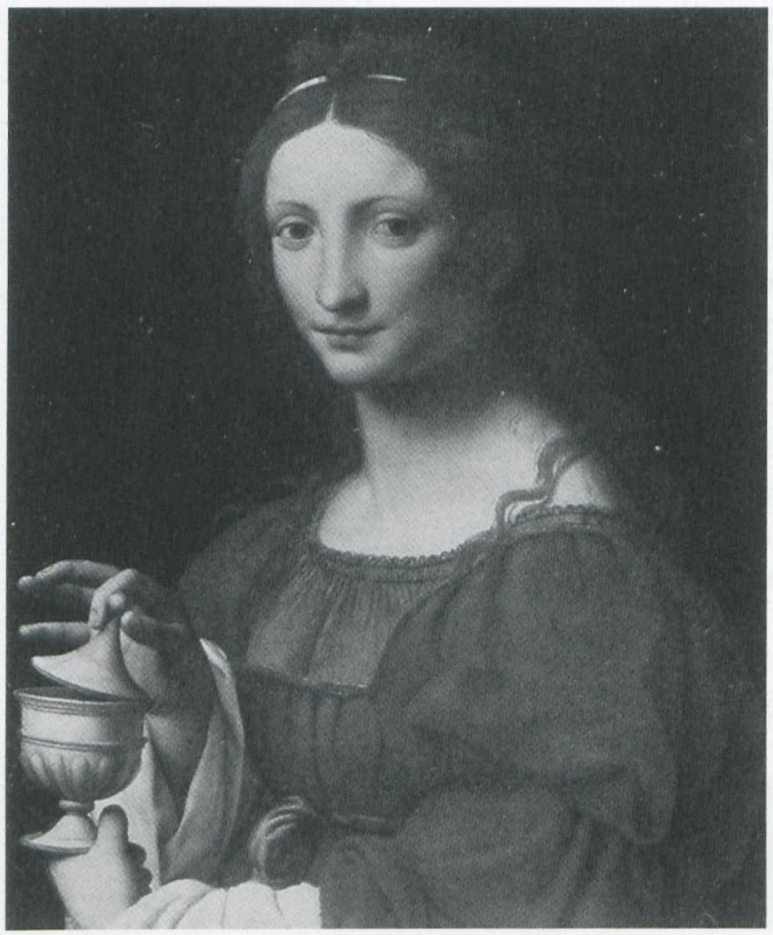

Abb. 14: Bernardino Luini, hl. Maria Magdalena;

Washington, National

Gallery of Art.

(Farbtafel 26)

unvermeidbar ist, wenn sich auch das Attribut verschieden lesen lässt. ${ }^{54}$ Der Konnex zu den Sebastians-Darstellungen im Porträttypus aus dem Umkreis Leonardos ist hier unübersehbar.

Zurück zu Savoldo. Mary Pardo hat in ihrem Aufsatz die beschriebenen Veränderungen der Berliner gegenüber der Londoner Fassung als Voraussetzung für die aktive Involvierung des Betrachters in den Akt der Sinnkonstitution des Bildes gedeutet. ${ }^{55}$ Das ist sicherlich zutreffend, aber was mich mehr als diese rezeptions-

54 Dies ist der Fall in dem Gemälde von Sebastiano del Piombo $(53 \times 46 \mathrm{~cm})$, das sich ebenfalls in der Washingtoner National Gallery of Art befindet. Es wurde von Luitpold Dussler noch als „Magdalena“" geführt (Luitpold Dussler, Sebastiano del Piombo, Basel 1942, S. 138, Nr. 44), mittlerweile gilt es recht durchgängig als Darstellung eines jungen Mädchens, da das Gefäß in ihrer Hand eher als ein Räucher-, denn als ein Salbgefäß anzusehen ist. Dies nimmt der Darstellung gleichwohl nichts von ihrer irritierenden Kraft, die durch die inschriftliche Bezeichnung „V. Colonna“ noch gesteigert ist. Vgl. Michael Hirst, Sebastiano del Piombo, Oxford 1981, S. 30 f. (A Wise Virgin); Ingenhoff-Danhäuser, a. O. Anm. 33, S. 42; Carlo Volpe/Mauro Lucco, Sebastiano del Piombo, Mailand 1980, S. 96, Nr. 18 (Ritratto di donna come vergine sacra).

55 Pardo, a. O. Anm. 34, S. 74: „By providing the Magdalene with a text (indeed a dramatic script), the beholder is empowered to decipher her pictorial message and breathe life into the fixity of the image. He becomes the painter's accomplice, but also his creature, assuming an essential role in the space between two fictions. Properly speaking, the 'subject' of Savoldo's Magdalene is as much the viewer as the saint or the Gospel narrative“. 
ästhetische Dimension der Bilder interessiert, ist der produktionsästhetische Aspekt an Savoldos Vorgehen. Schon im Londoner Gemälde, das allgemein als die früheste Version gilt, wird allein durch die höchst originelle Sujeterfindung, für die es, wie erwähnt, keine Bildtradition gibt, in Verbindung mit der ästhetischen Entscheidung für eine „storia“ in Halbfigur, nahegelegt, dass für den Maler bei der Erfindung dieses Sujets allein durch die spärlichen Attribute und die Neuartigkeit des Bildthemas ein wesentlicher Anreiz am Prozess der Signifikation bestanden haben muss. Besonders interessant ist, dass sich dies mit einem "dynamischen" oder prozessualen Moment verbindet, und zwar dadurch, dass Savoldo in den folgenden Fassungen an derselben Bildformulierung diesen Prozess der Bedeutungsstiftung noch einmal austestet und dabei eine wesentlich radikalere Lösung findet. Er lotet aus, wie viel an Bezeichnungsfaktoren er ins Bild setzen muss, damit ein Betrachter eine Benennung noch leisten kann, und ab wann das eigentlich nicht mehr zu gewährleisten ist - was im Berliner Gemälde zumindest für diejenigen Betrachter, die die Beziehung zu den übrigen Fassungen nicht herstellen können, anzunehmen ist. Der Prozess der Bildfindung ist hier also wesentlich ein Prozess der Reflexion über die Verweiskraft von Zeichen. Was sich in den genannten Darstellungen für Maler wie Giorgione, Boltraffio und Parmigianino andeutet, liegt hier durch die „sukzessive“ Arbeit in verschiedenen erhaltenen Bildfassungen offen zu Tage.

\section{Intendierte Ambiguität}

Wir werden also der Reflexion über die Darstellungskraft der Zeichen und der Relation von res und signa genau an der Stelle habhaft, wo ihr Auseinanderbrechen erprobt wird: So wenn Savoldo die Benennungsfaktoren derart weit reduziert, dass eine Bildformulierung für sich allein sprechen muss, obwohl sie das - wie im Berliner Bild - angesichts der Einzigartigkeit des Sujets eigentlich nicht kann, oder wenn in den Werken von Parmigianino, Sebastiano del Piombo und Giorgione nur ein Attribut eine Benennbarkeit gewährleistet, die aber von der Darstellungsweise der Figur eher unterlaufen wird - geht man im Fall von Giorgiones Bild hypothetisch davon aus, dass es sich bei dem Knaben mit Pfeil um einen Sebastian handeln soll oder das Gemälde zumindest diese Ikonographie als Möglichkeit aufruft. Genau an diesem Punkt zeichnet sich ein Decorum-Problem ab, das sich als Aufbrechen der Form-Inhalt-Adäquatheit manifestiert, weil der Kerngedanke des Decorum, dass ein bestimmtes Thema eine spezifische Darstellungsweise erfordert, hier seine Verbindlichkeit einbüßt. Ich möchte auch hier lieber von einem Ausloten der Grenzen einer Norm als einem veritablen Bruch sprechen, und gerade dieses Austesten führt zu einer Bewussthaltung der Norm. Dies sind Dinge, die in der Kunsttheorie nicht formuliert wurden, sondern nur im visuellen Medium erprobt wurden, was leicht damit zu begründen ist, dass die Theorie in dieser Zeit meist normativ argumentiert. Ich denke, dass in diesem weitgehenden Fehlen einer diskursiv-verbalen Reflexion über die Signifikation in kunsttheoretischen Schriften 


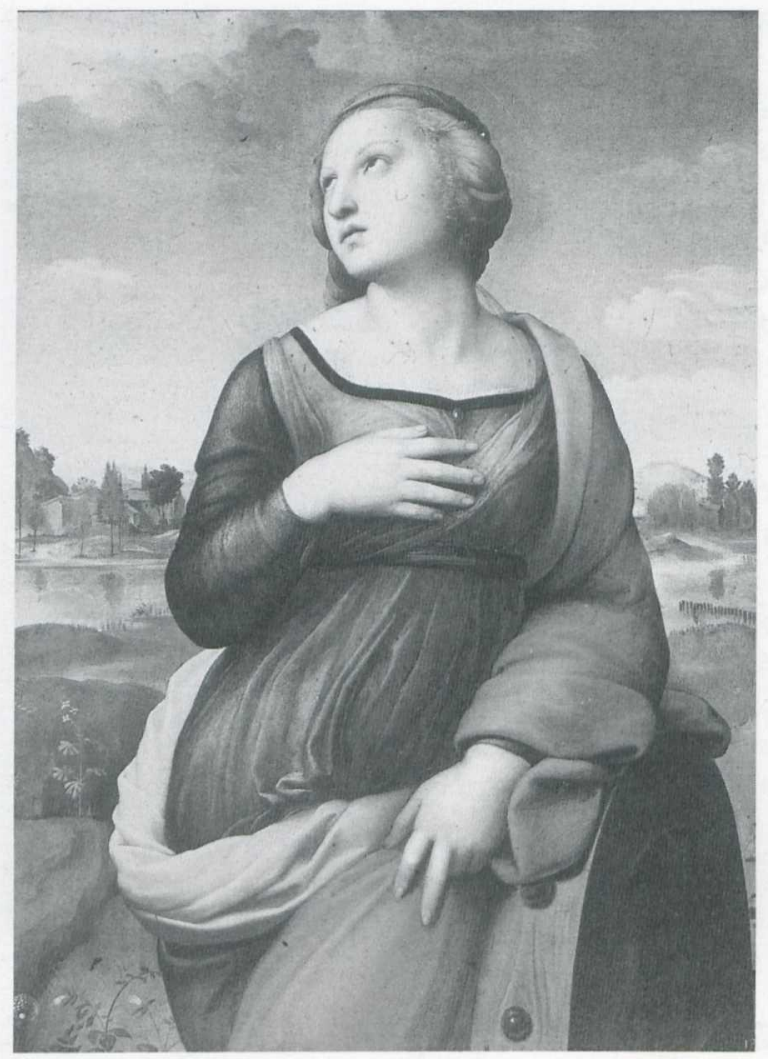

Abb. 15: Raffael,

hl. Katharina; London,

National Gallery.

(Farbtafel 27)

der Grund liegt, warum das Thema von der Forschung so wenig beachtet wurde. Da das Auseinanderbrechen von „res et signa“ durch Mehrfachbesetzung der Zeichen in der Theorie der Zeit nicht gedacht wird, scheint man es in der Forschung auch in der zeitgleichen Malerei nicht wahrnehmen zu wollen.

Um letzteren Gedanken zu belegen, sei auf ein Werk von Raffael hingewiesen, ein Künstler, der nun gerade nicht zu denjenigen zu zählen ist, über die wenig geschrieben wurde. Was allerdings in der umfangreichen Literatur über ihn nie zum Thema gemacht wurde, ist, dass es in Raffaels Gemälden einige äußerst exakte Formwiederholungen gibt, bei denen Gattung und Kontext der Figur geändert wird: So wiederholt Raffael beispielsweise die Serpentina-Haltung der hl. Katharina von Alexandrien in seinem berühmten Londoner Ölgemälde in Halbfigur (Abb. 15) ${ }^{56}$ mit nur geringfügigen Veränderungen, welche den Grad der Torsion und die Gestik betreffen, in seinem Fresko der Nymphe Galatea auf einer von Delphinen gezogenen Mu-

56 Holz; 71,5 x 55, 7cm; 1507/08; Meyer zur Capellen, a. O. Anm. 20, S. 269-272, Nr. 38; FerinoPagden, a. O. Anm. 20, S. 80, Nr. 51; Béguin/Garofalo, a. O. Anm. 20, S. 91, Nr. 44. Diesen Hinweis verdanke ich Christoph Thoenes. 
Abb. 16: Ders., Triumph der Galatea; Rom, Villa Farnesina.

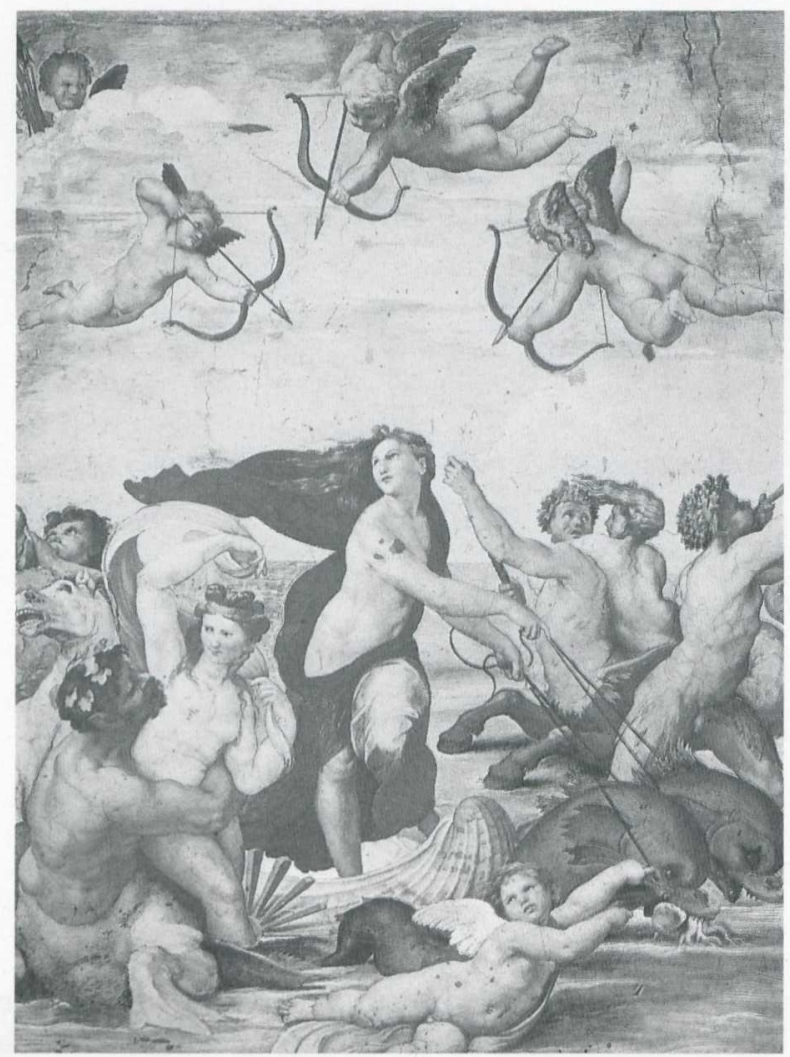

schel, das er vier Jahre später für die Villa des Bankiers Agostino Chigi in Trastevere ausführte (Abb. 16). ${ }^{57}$ Er ändert lediglich die Kleidung und die Frisur der Figur. Eine beliebig wiederholbare und mit je verschiedenem Inhalt zu füllende Form unterläuft aber die mit dem Decorum-Konzept verbundene Interdependenz von Form und Inhalt. In den genannten Beispielen folgt die Form nicht aus thematischen und wirkungsorientierten Vorgaben, sie wird vielmehr profan umbesetzt und so semantisch neu aufgeladen.

Eher pragmatisch motiviert, aber nichtsdestotrotz signifikant ist ein Formtransfer in Tizians Euvre, der jüngst publik gemacht wurde. ${ }^{58}$ Es handelt sich um eine halbfigurige Darstellung des hl. Tobias mit einem Engel, die erstmals in einem Inventar der Sammlung Barbarigo von 1750 dokumentiert ist (Abb. 17). Da Cri-

57 Fresko, 295 × 225 cm; 1511-13; Sala di Galatea; siehe Ferino-Pagden/Zancan, a. O. Anm. 20, S. 96, Nr. 59; Béguin/Garofalo, a. O. Anm. 20, S. 129, Nr. 61.

58 Jaynie Anderson, „Titian's unfinished portrait of a patrician woman and her daughter from the Barbarigo collection, Venice“, in: The Burlington Magazine 144 (2002), S. 671-679; Important old master pictures: evening sale. Christie, Manson \& Woods, London (7197): 8.12.2005, lot 54, S. 146-161. 


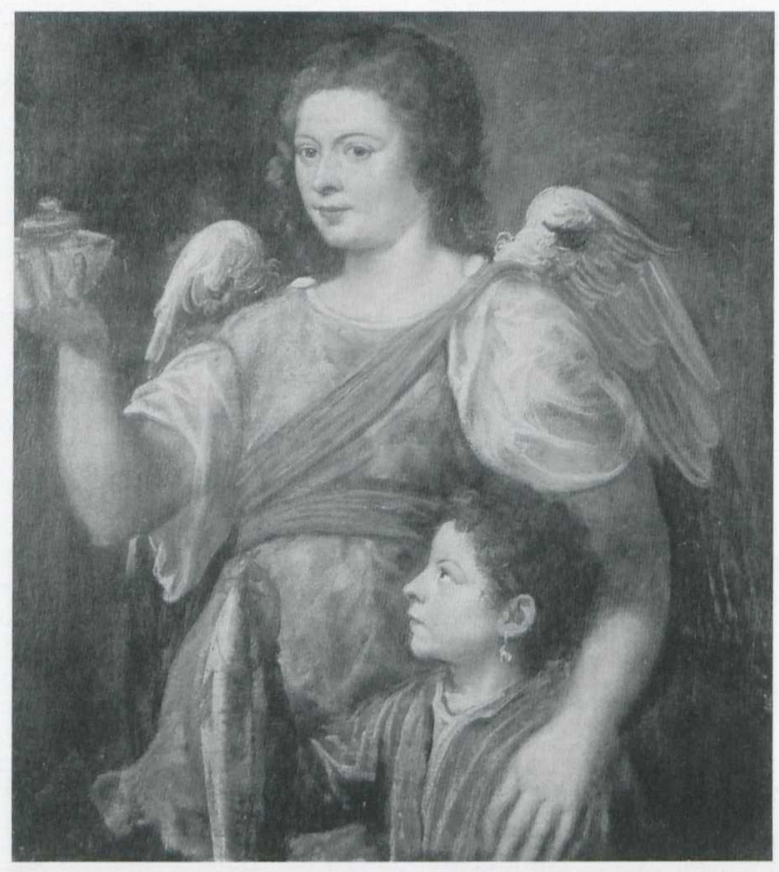

Abb. 17: Tizian, Doppelbildnis (Zustand vor Entfernung der Übermalungen); London, Kunsthandel. (Farbtafel 28)

stoforo Barbarigo den Nachlass des 1576 verstorbenen Künstlers erworben hatte, ist davon auszugehen, dass dieses Gemälde bei Tizians Tod unverkauft in der Werkstatt verblieben war. Die ästhetisch wenig überzeugende Relation des übergroßen Engels mit einem Gefäß in der erhobenen Rechten zu dem an seiner Seite stehenden hl. Tobias mit Ohrring (!) und einem großen Fisch in der Hand mag hierfür der Grund gewesen sein. Sie veranlasste wohl auch die jüngst erfolgte Restaurierung des Bildes, bei der in den tieferen Bildschichten eine im Formgerüst sehr ähnliche, in den Attributen, Kleidung und Äußerem der Figuren aber divergierende und ästhetisch ungleich überzeugende Darstellung freigelegt wurde (Abb. 18), in welcher auch die Beziehung der Figuren zueinander weitaus sinnvoller motiviert ist. Denn wir haben nun das Porträt einer Dame vor uns, die mit dem linken Arm ein kleines Mädchen umfasst. Vermutlich handelt es sich um ein Doppelbildnis von Mutter und Kind - ob tatsächlich von Angehörigen aus Tizians Familie, worauf der Verbleib des Bildes in Tizians Besitz hinweist, ist unsicher. ${ }^{59}$ Was Tizian dazu veranlasst hat, das Gemälde vermutlich durch einen Schüler oder Werkstattangehörigen ${ }^{60}$ partiell übermalen zu lassen, wodurch aus der Dame mit geflochtenen Haaren und einem Mädchen an ihrer Seite ein geflügelter Engel mit langen offenen Locken samt hl. Tobias wurde, ist unklar - vermutlich war es die

59 Important old master pictures 2005, S. 151.

60 Important old master pictures 2005, S. 149. 
Abb. 18: Ders., Doppelbildnis (Zustand nach Entfernung der Übermalungen). (Fabrtafel 29)

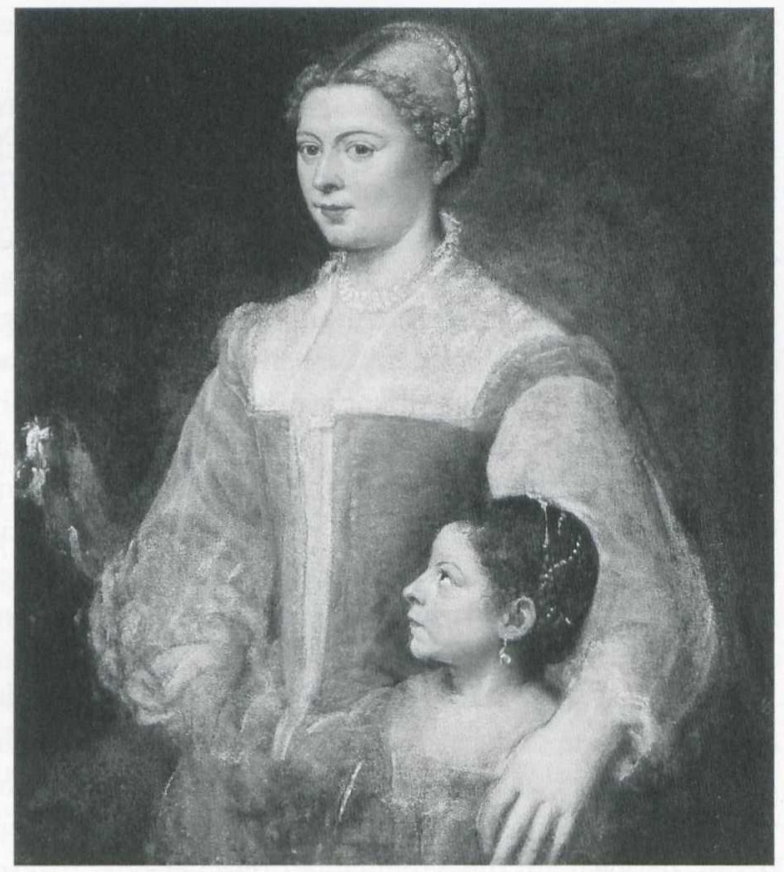

Nachfrage nach einem entsprechenden Sujet, das dann für den postulierten Interessenten möglicherweise nicht befriedigend umgesetzt worden ist, weshalb das Bild in der Werkstatt verblieb.

Dass Tizian "transformativ“ gearbeitet hat, also von einer einmal gefundenen Bildformulierung ausgehend etwas Neues geschaffen hat, ja dabei sogar auf dem Bildträger aus der einen eine andere Bildlösung entwickelt hat, ist uns mehrfach dokumentiert. ${ }^{61}$ Was im Kontext meiner Überlegungen an diesem Beispiel einer von einem Doppelporträt in eine Darstellung des hl. Tobias mit einem Engel verwandelten Gemäldes besonders interessant ist, ist die künstlerische Nonchalance, mit der er unter Änderung weniger Merkmale wie den offenen Locken und durch die einfache Hinzufügung von Attributen ein (profanes) Porträt einer Dame in eine sakrale Darstellung verwandelt. Auch wenn sich dies mit besonderen Werkstattbedingungen erklären mag, indiziert es eine Variabilität von Darstellungsweise und -sujet, die unter dem Vorzeichen einer Form-Inhalt-Interdependenz und -Korres-

61 So in seinem Spätwerk, in dem er großformatige Gemälde wie die Dornenkrönung Christi in München und die Schindung des Marsyas in Kremsier ausgehend von bzw. über (Kopien) eigener früherer Bildfassung entwickelte. Siehe hierfür, für weitere Beispiele sowie den kunsttheoretischen Kontext solcher Phänomene: Valeska von Rosen, Mimesis und Selbstbezüglichkeit in Werken Tizians. Studien zum venezianischen Malereidiskurs, Emsdetten/Berlin 2001, S. 398-422. 
pondenz, wie sie im rhetorischen Kunstsystem gefordert wurde und als Selbstverständlichkeit galt, beachtlich ist.

Was sind, abschließend gefragt, die Voraussetzungen für die beschriebenen Phänomene intendierter Ambiguität und bewusster Durchbrechungen der Form-Inhalt-Adäquanz? Sie liegen, wie mehrfach angedeutet, in der innerbildlichen Reflexion über die deiktische und bedeutungsgebende Funktion visueller Zeichen, konkret über das Verhältnis von res et signa. Es wird sozusagen ex negativo ausgetestet, wie bedeutungsgenerierend Attribute und Darstellungsweisen sind, indem durch das Minimieren eindeutiger Zeichen das potentielle und latente Auseinanderfallen von Signifikant und Signifikat erprobt wird. Die Gründe für diese Versuche sind sicherlich vielfältiger Natur. So dürfte gerade der Vorgang einer Kodifizierung von Wissen und Bildern in Enzyklopädien, Musterblättern und anderen Schriften im Cinquecento, in denen klare Zuordnungen von res und signa formuliert wurden, entsprechende Tendenzen ausgelöst oder zumindest verstärkt haben - ein Prozess „spielerischer" Gegenläufigkeit, mit dessen Dynamiken ich mich an anderer Stelle ausführlicher beschäftigt habe. ${ }^{62}$

Ein weiterer Grund wird in den gewandelten Funktionen und Kontexten solcher Werke zu suchen sein: Bilder werden gesammelt und im privaten Kontext nun auch unter neuen Bedingungen rezipiert. Ich habe diese Ebene hier bewusst vernachlässigt, um das signifikante Moment der Bilder nicht dadurch zu eskamotieren, dass ich auf die Möglichkeit pluraler oder diskrepanter Lektüren der Bilder abhebe. Selbstverständlich sind Wahrnehmungen niemals einheitlich, aber worauf es mir hier ankommt, ist, dass es bestimmte Merkmale der Bilder sind, die plurale Lektüren steuern, ja bedingen. Tatsächlich hängen ja produktions- und rezeptionsästhetische Prozesse unabdingbar miteinander zusammen. Dass solche Phänomene gleichwohl nur im Rahmen einer Betrachter- und Gesprächskultur möglich sind, deren Mitglieder dazu bereit sind, sie gedanklich nachzuvollziehen, ja mehr noch, an ihnen Gefallen zu finden und über sie zu „ragionare“, versteht sich. ${ }^{63}$ Es ist der Betrachter, der von ambigen Gemälden in neuer Weise aktiviert und gefordert wird, Relationen von Signifikanten und Signifikaten herzustellen und dem Prozess des Entzugs der Eindeutigkeit einerseits und der Bedeutungskonstitution im Akt der Wahrnehmung andererseits nachzuspüren. Dass auch gerade diese funktionale und rezeptionsästhetische Ebene eine entscheidende Voraussetzung beim Entstehen ambiger Bilder hat, wird noch wahrscheinlicher dadurch, dass diese Tendenz im frühen Seicento, in dem vermehrt Sammlungen entstehen, ungleich dominanter wird. ${ }^{64}$

62 Valeska von Rosen, Caravaggio und die Grenzen des Darstellbaren. Ambiguität, Ironie und Performativität in der Malerei um 1600, Berlin 2009.

63 Zur Gesprächskultur Venedigs siehe von Rosen, a. O. Anm. 61, S. 81-139 (mit weiterer Literatur).

64 Hierzu ausführlicher von Rosen, a. O. Anm. 62. 


\section{Literatur}

Alberti, Leone Battista, Della pittura. Über die Malkunst, hrsg., eingel., übers. u. komm. v. Oskar Bätschmann, Sandra Gianfreda, Darmstadt 2002.

Anderson, Jaynie, „Titian's unfinished portrait of a patrician woman and her daughter from the Barbarigo collection, Venice", in: The Burlington Magazine 144 (2002), S. 671-679.

Arasse, Daniel, Leonardo da Vinci, Köln 1999, S. 461-473.

Baldriga, Irene, „Raffaello Sanzio. Saint Sébastien“, in: Patrizia Nitti/Marc Restellini (Hrsg.), Raphaël. Grace et Beauté (Ausst.-Kat. Paris 2001/2002), Paris 2001, S. 82-85.

Béguin Sylvie/Garofalo, Cristiana, Raffaello. Catalogo completo dei dipinti, Santarcangelo di Romagna 2002.

Boehm, Gottfried (Hrsg.), Was ist ein Bild? München ${ }^{2} 1995$.

Bohde, Daniela, „Ein Heiliger der Sodomiten? Das erotische Bild des HI. Sebastian im Cinquecento", in: Mechthild Fend/Marianne Koos (Hrsg.), Männlichkeit im Blick: Visuelle Inszenierungen in der Kunst seit der Frühen Neuzeit, Köln 2004, S. 79-98.

Bohde, Daniela, Haut, Fleisch und Farbe. Körperlichkeit und Materialität in den Gemälden Tizians, Emsdetten/Berlin 2002.

Bruno, Giordano, Explicatio tringinta sigillorum, Opera latine conscripta, hrsg. v. F. Fiorentino u. a., Neapel/Florenz 1879-91, Facs.-Neudr. Stuttgart 1962.

Burnett, Ron, How Images Think, Cambridge (MA) 2004.

Büttner, Frank, „Rationalisierung der Mimesis: Anfänge der konstruierten Perspektive bei Brunelleschi und Alberti“, in: Andreas Kablitz/Gerhard Neumann (Hrsg.), Mimesis und Simulation, Freiburg 1998, S. 55-87.

Cancik, Hubert/Schneider, Helmuth (Hrsg.), Der Neue Pauly. Enzyklopädie der Antike, Stuttgart 1997.

Capellen, Jürg Meyer zur, Raphael. A critical catalogue of his paintings. I The Beginnings in Umbria and Florence, ca. 1500-1508, Landshut 2001.

Chiara, Piero/Alberto Dell'Acqua, Gian u. a. (Hrsg.), Sacro e profano nella pittura di Bernardino Luini (Ausst.-Kat. Varese 1975) Cinisello Balsamo/Mailand 1975.

Cremonini, Claudia, „Le raccolte d'arte del Cardinale Alessandro d'Este. Vicende collezionistiche tra Modena e Roma“, in: Jadranka Bentini (Hrsg.), Sovrane passioni. Studi sul collezionismo estense, Mailand 1998, S. 91-137.

Damisch, Hubert, L'origine de la perspective, Paris ${ }^{3} 1993$.

Darriulat, Jacques, Sebastien. Le Renaissant. Sur le martyre de saint Sébastien dans la deuxième moitié du Quattrocento, Paris 1998.

Dussler, Luitpold, Sebastiano del Piombo, Basel 1942.

Ferino-Pagden, Sylvia, in: Lucia Fornati/dies. (Hrsg.), Parmigianino und der europäische Manierismus (Ausst.-Kat. Parma/Wien 2003), Cinisello Balsamo 2003.

Ferino-Pagden, Sylvia/Zancan, M. Antonietta, Raffaello. Catalogo completo dei dipinti, Florenz 1989.

Fiorio, Maria Teresa, Giovanni Anontio Boltraffio. Un pittore milanese nel lume di Leonardo, Mailand/Rom 2000.

Fomiciova, Tamara, „Giorgione e la formazione della pittura di genere nell'arte veneziana del XVI secolo", in: Giorgione. Atti del convegno Internazionale di Studio per il $5^{\circ}$ Centenario della nascita (Castelfranco Veneto 1978), Venedig 1979, S. 159-164.

Foucault, Michel, Les mots et les choses, Paris 1966.

Frangi, Francesco, „Giampetrino“, in: Mina Gregori (Hrsg.), Pittura a Milano. Rinascimento e Manierismo, Mailand 1998, S. 243-245. 
Freedman, Luba, „Saint Sebastian in Veneto Painting: The ,Signals' Addressed to ,Learned Spectators", in: Venezia Cinquecento 15 (1988), S. 5-20.

Frimmel, Theodor, Der Anonimo Morelliano. Marcantonio Michel's Notizia d'opere del disegno, Wien 1888.

Gilbert, Creighton, The Works of G. Savoldo, Diss. 1955.

Gilbert, Creighton, The Works of Girolamo Savoldo. The 1955 Dissertation with a Review of Research 1955-1985, New York/London 1986.

Gould, Cecil, National Gallery Catalogues. The Sixteenth-Century Italian Schools, London 1975

Hadeln, Detlev von, Die wichtigsten Darstellungsformen des h. [!] Sebastian in der italienischen Malerei bis zum Ausgang des Quattrocento, Straßburg 1906.

Haskins, Susan, Mary Magdalen, Myth and Metaphor, London 1993.

Henkel, Arthur/Schöne, Albrecht (Hrsg.), Emblemata. Handbuch zur Sinnbildkunst des XVI. und XVII. Jahrhunderts, Stuttgart 1967.

Hirst, Michael, Sebastiano del Piombo, Oxford 1981.

Homann, Holger, Studien zur Emblematik des 16. Jahrhunderts, Utrecht 1971.

Hornig, Christian, Giorgiones Spätwerk, München 1987.

Important old master pictures: evening sale. Christie, Manson \& Woods, London (7197).

Ingenhoff-Danhäuser, Monika, Maria Magdalena. Heilige und Sünderin in der italienischen Renaissance. Studien zur Ikonographie der Heiligen von Leonardo bis Tizian, Tübingen 1984.

Koos, Marianne, „Amore dolce-amaro. Giorgione und das ideale Knabenbildnis der venezianischen Renaissancemalerei“, in: Marburger Jahrbuch für Kunstwissenschaft 33 (2006), S. 113-174.

Koos, Marianne, „Giorgione da Castelfranco. Knabe mit Pfeil“, in: Sylvia Ferino-Pagden/ Giovanna Nepi Scirè (Hrsg.), Giorgione. Mythos und Enigma (Ausst.-Kat. Wien 2004), Mailand 2004, S. 184-187.

Koos, Marianne, Bildnisse des Begehrens. Das lyrische Männerporträt in der venezianischen Malerei des frühen 16. Jahrhunderts - Giorgione, Tizian und ihr Umkreis, Emsdetten/ Berlin 2006.

Kraehling, Victor, Saint Sébastien dans l'art, Paris 1938.

Krüger, Klaus, Das Bild als Schleier des Unsichtbaren. Ästhetische Illusion in der Kunst der frühen Neuzeit in Italien, München 2001.

Maar, Christa/Burda, Hubert (Hrsg.), Iconic Turn. Die neue Macht der Bilder, Köln 2004.

Maisch, Ingrid, Maria Magdalena, Freiburg u. a. 1996.

Marani, Pietro C., „Giovan Pietro Rizzoli, called Giampetrino: Milan, documented from c. 1495 to 1549 “, in: The Legacy of Leonardo. Painters in Lombardy 1490-1530, mit Essays v. Giulio Bora u. a, Mailand 1998, S. 275-300.

Mosco, Marilena (Hrsg.), La Maddalena tra sacro e profano. Da Giotto a De Chirico (Ausst.Kat. Florenz), Florenz 1986.

Ottino Della Chiesa, Angela, Bernardino Luini, Novara 1956.

Otto, Stephan, „Rhetorische Techne oder Philosophie sprachlicher Darstellungskraft?“, in: Zeitschrift für philosophische Forschung 37 (1983), S. 497-514.

Otto, Stephan, Das Wissen des Ähnlichen. Michel Foucault und die Renaissance, Frankfurt a. M. 1992.

Otto, Stephan, Giambattista Vico, Grundzüge seiner Philosophie, Stuttgart 1989.

Otto, Stephan, Renaissance und frühe Neuzeit (Geschichte der Philosophie in Text und Darstellung 3), Stuttgart 1984. 
Pardo, Mary, „The Subject of Savoldo's Magdalene“, in: The Art Bulletin 71 (1989), S. $67-$ 91.

Pedretti, Carlo, Leonardo. A Study in Chronology and Style, London 1973.

Penny, Nicholas, National Gallery Catalogues. The Sixteenth Century Italian Paintings. I Paintings from Bergamo, Brescia and Cremona, London 2004.

Pignatti, Terisio/Pedrocco, Filippo, Giorgione, Mailand 1999.

Pozzolo, E. M. Dal, „Giovanni Antonio Boltraffio“, in: Saur Allgemeines Künstlerlexikon, XII, München/Leipzig 1996.

Praz, Mario, Studies in Seventeenth Century Imagery, London 1939.

Rama, Elena, „Un tentativo di rilettura della ritrattistica di Boltraffio fra Quatrocento e Cinquecento“, in: Arte Lombarda 64 (1983), S. 79-92.

Ressouni-Demigneux, Karim, Saint-Sébastien, Paris 2000.

Ridolfi, Carlo, Le Maraviglie dell'arte ouuero le uite degli illustri pittori ueneti e delo stato, Venedig 1648, hrsg. \& komm. v. Detlev Frhr. v. Hadeln, Berlin 1914.

Roscher, W. H. (Hrsg.), Ausführliches Lexikon der griechischen und römischen Mythologie, Leipzig 1884-86.

Rosen, Valeska von, Caravaggio und die Grenzen des Darstellbaren. Ambiguität, Ironie und Performativität in der Malerei um 1600, Berlin 2009.

Rosen, Valeska von, Mimesis und Selbstbezüglichkeit in Werken Tizians. Studien zum venezianischen Malereidiskurs, Emsdetten/Berlin 2001.

Rosen, Valeska von/Krüger, Klaus/Preimesberger, Rudolf (Hrsg.), Der stumme Diskurs der Bilder. Reflexionsformen des Ästhetischen in der Kunst der frühen Neuzeit, München/Berlin 2003.

Rossi, Ottavio, Elogi historici di bresciani illustri, Brescia 1620.

Schade, Karl, Andachtsbild. Die Geschichte eines kunsthistorischen Begriffs, Weimar 1996.

Schleier, Erich, „Savoldos Magdalena in der Berliner Gemäldegalerie. Zur Wiederherstellung eines Meisterwerks der Brescianer Cinquecentomalerei“, in: Jahrbuch der Berliner Museen 33 (1991), S. 135-147.

Schmidt-Biggemann, Wilhelm, „Topische Modelle in Theorie und Praxis der Renaissance“, in: Ulrich Pfisterer/Max Seidel (Hrsg.), Visuelle Topoi. Erfindung und tradiertes Wissen in den Künsten der italienischen Renaissance, München/Berlin 2003, S. 11-20.

Scholz, Bernhard F., Emblem und Emblempoetik: Historische und systematische Studien, Berlin 2002.

Sebastiaan. Martelaar of mythe, Zwolle 1993.

Seilern, Antoine, Italian Paintings and Drawings at 56 Princes Gate London SW7, London 1959.

Settis, Salvatore, Giorgiones, Gewitter. Auftraggeber und verborgenes Sujet eines Bildes in der Renaissance, Berlin 1982.

Shell Janice/Sironi, Grazioso, „Some documents for Giovanni Pietro Rizzoli: il Giampetrino“, in: Raccolta Vinciana 25 (1993), S. 121-135.

Shell, Janice, „Marco d'Oggiono: Oggiono, c. 1475 - c. 1530“, in: The Legacy of Leonardo. Painters in Lombardy 1490-1530, mit Essays v. Giulio Bora u. a, Mailand 1998, S. 163178.

Sitt, Martina, „Meister der weiblichen Halbfiguren“, in: Alte Rabmen - neue Sicht (=Hefte der Hamburger Kunsthalle), Hamburg 2000, o. Pag.

Spagnolo, Maddalena, "Correggio's Reclining Magdalen. Isabella d'Este and the Cult of St Mary Magdalen“, in: Apollo 157 (2003), Nr. 496, S. 37-45.

Staatliche Museen Preußischer Kulturbesitz, Berlin, Gemäldegalerie. Katalog der ausgestellten Werke des 13.-18. Jahrhunderts, Berlin 1975. 
Stradiotti, Renata in: Sybille Ebert-Schifferer (Hrsg.), Giovanni Gerolamo Savoldo und die Renaissance zwischen Lombardei und Venetien. Von Foppa und Giorgione bis Caravaggio (Ausst.-Kat. Frankfurt/Brescia 1990), Mailand 1990, S. 140-147.

Suckale, Robert, „Arma Christi. Überlegungen zur Zeichenhaftigkeit mittelalterlicher Andachtsbilder", in: Städel-Jahrbuch 6 (1977), S. 177-208.

Suckale, Robert, Stil und Funktion. Ausgewählte Schriften zur Kunst des Mittelalters, hrsg. v. Peter Schmidt, Gregor Wedekind, München/Berlin 2003.

Suida, Wilhelm, Leonardo und sein Kreis, München 1929.

Vaccaro, Mary, Parmigianino: i dipinti, Turin 2002.

Valla, Lorenzo, „Dialecticae disputationes“, in: Opera omnia, hg. u. eingel. v. Eugenio Garin, Turin 1962.

Vecellio, Cesare, Habiti antichi et moderni di tutto il mondo, Venedig 1598.

Volpe, Carlo/Lucco, Mauro, Sebastiano del Piombo, Mailand 1980. 